\title{
Identification, Evaluation and Prioritization of Human Errors in the Nursing Population of the Hospital's Coronary Care Unit
}

Amir Abbasi garmaroudi, MSc, Department of Occupational Health Engineering and Students' Scientific Research Center, School of Public Health, Tehran University of Medical Sciences, Tehran, Iran.

(1) Sajjad Mozaffari, ( ${ }^{\star}$ Corresponding author), MSc, Department of Occupational Health Engineering and Students' Scientific Research Center, School of Public Health, Tehran University of Medical Sciences, Tehran, Iran. smozaffari@razi.tums.ac.ir

Mahshid Bahrami, MSc, Department of Occupational Health Engineering and Students' Scientific Research Center, School of Public Health, Tehran University of Medical Sciences, Tehran, Iran.

Hadi Alimoradi, MSc, Department of Occupational Health Engineering, School of Public Health, Yazd University of Medical Sciences, Yazd, Iran.

\section{Abstract}

Background and aims: Every year, many patients in different wards of the hospital die due to human errors. In the field of human errors related to clinical care, one of the most important aims is to identify and prevent the occurrence of harmful effects caused by such errors. Thus, in order to prevent human errors, one of the preventive approaches is to recognize and analyze them. The purpose of this study is, to investigate all clinical care processes in the coronary care unit, identify human errors and prioritize the tasks and errors in this section.

Methods: This descriptive-analytical study was performed cross-sectional in three stages. In the first stage, human errors were identified and evaluated using the SHERPA approach, and the frequency of each human error in the CCU was determined. In the second stage, the criteria and factors affecting the occurrence of human error in the CCU were determined by Delphi method. Finally, in the third stage, with the Analytic hierarchy process, the criteria and tasks that had the highest frequency of errors, were prioritized. SPSS. 24 and Expert choice. 11 are the two softwares that were used to analyze the data in this study.

Results: Among the 116 identified errors with SHERPA, the highest percentages of identified errors among all the errors, were related to functional errors $(68.1 \%)$ and the lowest percentages was related to selective errors $(2.58 \%)$, respectively. The results of different Delphi rounds showed that the factor of skill, experience, equipment, work time and workload are the most important factors that affect the occurrence of human errors in CCU. By pair comparison of this factors, we found that the experience and skill of the nurses, with a weight of 0.278 and 0.272 , was the most important, and the equipment's factor with a weight of 0.087 , was the least important among the five effective factors for the incidence of human errors in CCU. The task of extracting airway discharge with a weight of 0.125 , was the most preferred among 16 tasks, in other words, the task of extracting the airway discharge had the most risk for error amongst all the functional errors. The factor of experience and skill, was the most important factor in the occurrence of functional errors. In the error type of recovery, the task of identifying the drug information (time, date, dose and dosage form, etc.) with a weight of 0.054 , had the highest rank among this type of errors. The factor of experience and loading with weights of 0.018 and 0.01 , were the most important among this type, respectively.

Conclusion: In the coronary care unit, the functional and revision errors in the vital tasks such as cardiopulmonary resuscitation, dose adjustment, and the lack of proper drug injection, were the most common errors among all. Therefore, design and the implementation of control measures such as periodic trainings on how to do the job properly, preparation of checklists with a focus on human behavior in different job processes, is recommended to eliminate or reduce the amount of identified errors in the hospital's CCU.

Conflicts of interest: None

Funding: None

\author{
Keywords \\ SHERPA \\ Human error \\ CCU \\ Hospital \\ Analytic hierarchy process
}

Received: 2019/06/03

Accepted : 2020/06/25 


\section{INTRODUCTION}

Based on studies in the field of industrial accidents, the human factor has been reported as the most important factor in the occurrence of accidents (1). By definition, human error is any deviation of human performance from the rules and duties, in a way that impacts the efficiency of the system adversely. According to reports, human errors have been responsible for more than 90 percent of the accidents, such as Fleigsburg in the British chemical industry and Chernobyl in the Russian nuclear reactor (2). Medical errors are divided into three categories, as follows: the preventable $(70 \%)$, probably preventable $(6 \%)$ and unpreventable $(4 \%)$ medical errors based on their frequency, respectively (3). According to the medical statistics from the United States in 1999, 44,000 to 98,000 deaths, and more than one million injuries were reported each year, due to such errors. One of the preventive approaches in prevention of human errors, is to analyze them. In the present study, the Delphi method and the Analytical Hierarchy Process (AHP) were used, to determine the affecting factors on the occurrence of human errors and to prioritize the tasks that were most at risk by the human error. The invention of the AHP method, is one of the most widely used multi-factor decision models. Delphi method, as a scientific tool to collect the necessary information to assess the safety risk is recommended (4). According to research, $4 \%$ of the hospitalized patients, suffer from injuries. More than $70 \%$ of these injuries are preventable (5). In the research of Jafarvand et al, which was conducted with the aim of identifying and evaluating human errors by SHERPA method, to reduce human errors, the control strategies such as using individual with sufficient skills and experiences is recommended (5). Considering the high efficiency of the SHERPA method in identifying human errors in medical centers, the aim of this study is to identify and evaluate human errors, also to prioritize tasks by considering the affecting criteria of human error in the Intensive Care Unit (ICU) by using Delphi method and Analytical Hierarchy Process (AHP).

\section{METHODOLOGY}

This descriptive-analytical study was performed as cross-sectional, to identify the human errors of the Coronary Care Unit's (CCU) nurses. From the population of $100 \mathrm{~N}$ coronary care unit nurses in five hospitals that are located in Tehran, 10 people were randomly selected ( 5 women and 5 men) to investigate the human errors. The age range of the subjects was 40 to 45 years; they did not have any underlying disease.

In the first stage of SHERPA, the tasks were divided into five categories (functional, retrieval, checking, selection, and communication), and finally, the identified errors due to tasks were coded based on SHERPA. In the second stage, the affecting criteria on the human errors in the CCU were extracted. In the third stage, in order to weight and prioritize the studied tasks, the Analytic Hierarchy Process (AHP) was used. In the first level, decision making is targeted, and in the second level, indicators (criteria) and in the third level, options (tasks) are prioritized. The priority of tasks was scored from 1 to 9, based on the Likert scale. The Delphi was available for a group of experts, which was done by distributing questionnaires among these people (10). In the second stage, the affecting criteria on the human errors in the CCU were extracted and is exhibited in (Table 1). In this study, the Delphi group consisted of 10 university professors, $\mathrm{PhD}$ students in ergonomics and occupational health engineering. Finally, the various rounds of Delphi were performed as follows:

1. Requesting from the Delphi group in the first

Table 1. List of Affecting Criteria on Human Error in The CCU (Extracted from The Delphi Method and Research Background)

\begin{tabular}{lllll}
\hline Communicational & Retrieval & Selective & Checking & Functional \\
\hline Equipment & Motivation & Skill & work condition & Poor communication \\
communication & Education & Experience & Management & security issues \\
Fatigue & Equipment & Education & Amount of received salary & Lack of time to do work \\
Education & Condition & workload & Education & Organizational policy \\
Experience & Equipment & Accuracy & Equipment & Equipment \\
Skill & Skill & Awareness & System error & The device is not \\
Repetitive work & Instruction & information & Work protocol & work condition \\
& Education & work condition & Period & Education \\
& Fatigue & Transparency of & Knowledge & Skill \\
& Workload & responsibility & Skill & Experience \\
\hline
\end{tabular}


round to introduce and propose the criteria related to the occurrence of various types of human errors in the CCU.

2. Scoring the degree of relevance of the obtained criteria from the first round and the research background by using a questionnaire (scoring is from 1 to 10 , and the criterion that can get average seven or more, was considered among the most important criteria.).

3. To eliminate the criteria that did not get the required score and then resubmit a new questionnaire to re-score the remaining criteria.

4. Performing criteria screening operations and calculating the incompatibility coefficient, to ensure the coordination of opinions and ending the Delphi rounds (otherwise, performing subsequent rounds)

In the third stage, in order to weight and prioritize the studied tasks, the Analytic Hierarchy Process (AHP) was used. In the first level, making-decision was targeted, and in the second level, indicators (criteria) and in the third level, options (tasks) are prioritized. The priority of tasks were scored from 1 to 9, based on the Likert scale.

\section{RESULTS}

The obtained results from the identification and evaluation of the human errors with the help of SHERPA, showed that there are 16 tasks for CCU nurses from the time of the patient's admission to the end of the treatment, which were accounted for the most number of errors. In total, 116 potential errors were identified. The most and least percentages of the detected errors from total errors, are related to the functional errors $(68.1 \%)$ and selective errors $(2.58 \%)$, respectively. Also, results showed that in the functional errors of CCU, the task of extracting the airways secretions with a weight of 0.125 , was the most preferred, and also showed that the experience and skill criteria with a weight of 0.035 , are the most

Table 2. Weight (Priority) of Tasks by Criteria Separation

\begin{tabular}{|c|c|c|c|c|c|c|c|c|c|}
\hline $\begin{array}{l}\text { Error } \\
\text { type }\end{array}$ & Task & Skill & $\begin{array}{l}\text { Work- } \\
\text { load }\end{array}$ & Experience & Equipment & Period & $\begin{array}{l}\text { Weight } \\
\text { relative } \\
\text { to target }\end{array}$ & $\begin{array}{l}\text { Indicator } \\
\text { criteria }\end{array}$ & Weight \\
\hline \multirow{9}{*}{ 胥 } & $\begin{array}{l}\text { Performing initial CPR } \\
\text { procedures }\end{array}$ & 0.022 & 0.031 & 0.046 & 0.007 & 0.018 & 0.124 & Experience & 0.046 \\
\hline & $\begin{array}{l}\text { Do piping in } \\
\text { emergency condition }\end{array}$ & 0.027 & 0.018 & 0.025 & 0.011 & 0.016 & 0.098 & Skill & 0.027 \\
\hline & $\begin{array}{l}\text { Immediate actions for } \\
\text { dysrhythmia }\end{array}$ & 0.024 & 0.017 & 0.019 & 0.008 & 0.017 & 0.085 & Skill & 0.024 \\
\hline & $\begin{array}{l}\text { Recording data in the } \\
\text { integrated hospital system }\end{array}$ & 0.013 & 0.013 & 0.016 & 0.005 & 0.010 & 0.056 & Experience & 0.016 \\
\hline & Exhale airways secretions & 0.035 & 0.022 & 0.035 & 0.012 & 0.021 & 0.125 & $\begin{array}{l}\text { Skill and } \\
\text { Experience }\end{array}$ & 0.035 \\
\hline & $\begin{array}{l}\text { Performing intramuscular } \\
\text { and intravenous injections }\end{array}$ & 0.023 & 0.013 & 0.016 & 0.005 & 0.013 & 0.070 & Skill & 0.023 \\
\hline & connecting serum set & 0.01 & 0.014 & 0.01 & 0.004 & 0.009 & 0.051 & Workload & 0.014 \\
\hline & Injection pump installation & 0.018 & 0.017 & 0.027 & 0.013 & 0.014 & 0.090 & Experience & 0.027 \\
\hline & Recording drug information & 0.009 & 0.010 & 0.018 & 0.004 & 0.009 & 0.053 & Experience & 0.018 \\
\hline \multirow{2}{*}{ 里 } & Patient identification & 0.008 & 0.008 & 0.009 & 0.003 & 0.006 & 0.034 & Experience & 0.009 \\
\hline & Heart monitoring & 0.014 & 0.007 & 0.009 & 0.003 & 0.006 & 0.039 & Skill & 0.014 \\
\hline \multirow{3}{*}{ 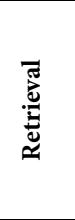 } & Patient identification & 0.008 & 0.008 & 0.009 & 0.003 & 0.006 & 0.034 & Experience & 0.009 \\
\hline & Reading the dose of drug & 0.017 & 0.006 & 0.013 & 0.003 & 0.007 & 0.046 & $\begin{array}{l}\text { Skill and } \\
\text { Experience }\end{array}$ & 0.015 \\
\hline & $\begin{array}{l}\text { Identification of drug } \\
\text { information (time, date, form } \\
\text { (of use, etc. }\end{array}$ & 0.022 & 0.010 & 0.012 & 0.003 & 0.007 & 0.054 & $\begin{array}{l}\text { Skill and } \\
\text { Experience }\end{array}$ & 0.017 \\
\hline 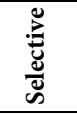 & $\begin{array}{l}\text { Selecting, adjusting and } \\
\text { applying DC shock }\end{array}$ & 0.007 & 0.006 & 0.007 & 0.002 & 0.004 & 0.027 & $\begin{array}{l}\text { Skill and } \\
\text { Experience }\end{array}$ & 0.007 \\
\hline \multirow{2}{*}{ 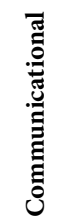 } & $\begin{array}{l}\text { Training before, during and } \\
\text { after hospitalization }\end{array}$ & 0.009 & 0.005 & 0.007 & 0.002 & 0.004 & 0.027 & Skill & 0.009 \\
\hline & $\begin{array}{l}\text { To Know the start code of } \\
\text { early stages from } \\
\text { cardiovascular resuscitation }\end{array}$ & 0.005 & 0.004 & 0.006 & 0.002 & 0.004 & 0.021 & Experience & 0.006 \\
\hline
\end{tabular}


important in performing this task correctly. The task of knowing the code and starting the initial stages of cardiovascular resuscitation with a weight of 0.021 , showed the lowest priority, so that in this task, the experience criterion with a weight of 0.006 , indicates the most importance in the correct implement of this task (Table 2).

\section{DISCUSSION}

To identify the most important criteria in the occurrence of human errors, by using the Delphi Group the data was analyzed, and it was determined that the five criteria; experience, skill, workload, time and equipment were the most important criteria in the CCU. The results of the paired comparisons between the criteria by using AHP, showed that the criteria of experience and skill with a weight of 0.278 and 0.272 , respectively, were allocated the most important, this result is in accordance with the studies of Stroud et al. (6), Dovey et al. (7). The task of connecting the serum set, allocated the lowest priority with a weight of 0.051 among the functional errors of this section, that work load criterion with a weight of 0.014 was the most important parameter for the error occurrence in performing this task. Garosi et al., by designing a serum set connector tract, somewhat reduced the workload of this task (8). In checking errors, the experience criterion with a weight of 0.006 , indicates the most importance in the correct implementation of the task from knowing the starting code of the early stages of cardiovascular resuscitation.

\section{CONCLUSION}

In the coronary care unit, the functional and revision errors in the vital tasks, such as cardiopulmonary resuscitation, dose adjustment, and proper drug injection were the most common. Therefore, the design and implementation of control measures such as periodic training on how to do the job properly, preparation of checklists with a focus on human behavior in different job processes is recommended to eliminate or reduce the amount of the identified errors in the hospital's CCU. Considering the importance of the human errors' consequences, and according to the type and percentage of the identified errors, with regard to evaluation and prioritization of the human errors in this study, can help to present and implement control strategies and corrective actions to eliminate or reduce the risk of human errors.

\section{ACKNOWLEDGMENT}

The authors of the article are extremely grateful to all the nurses and physicians who were involved in collecting and completing the information.

\section{CONFLICT OF INTEREST}

The authors declare that there are no conflicts of interest regarding the publication of this manuscript.

How to cite this article:

Amir Abbasi garmaroudi, Sajjad Mozaffari, Mahshid Bahrami, Hadi Alimoradi Identification. Evaluation and Prioritization of Human Errors in the Nursing Population of the Hospital's Coronary Care Unit. Iran Occupational Health. 2021 (01 June); 18:11.

*This work is published under CC BY-NC 4.0 licence 


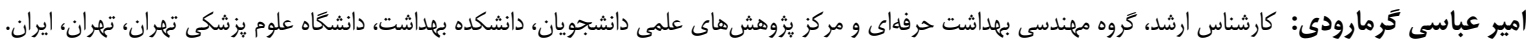

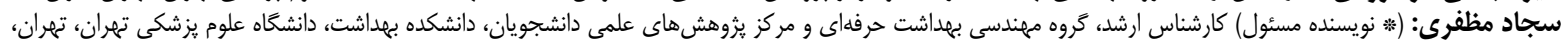
ايران. smozaffari@razi.tums.ac.ir

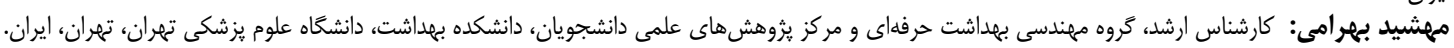

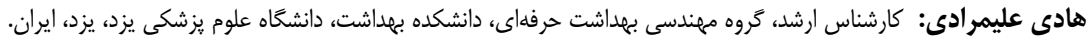

جك

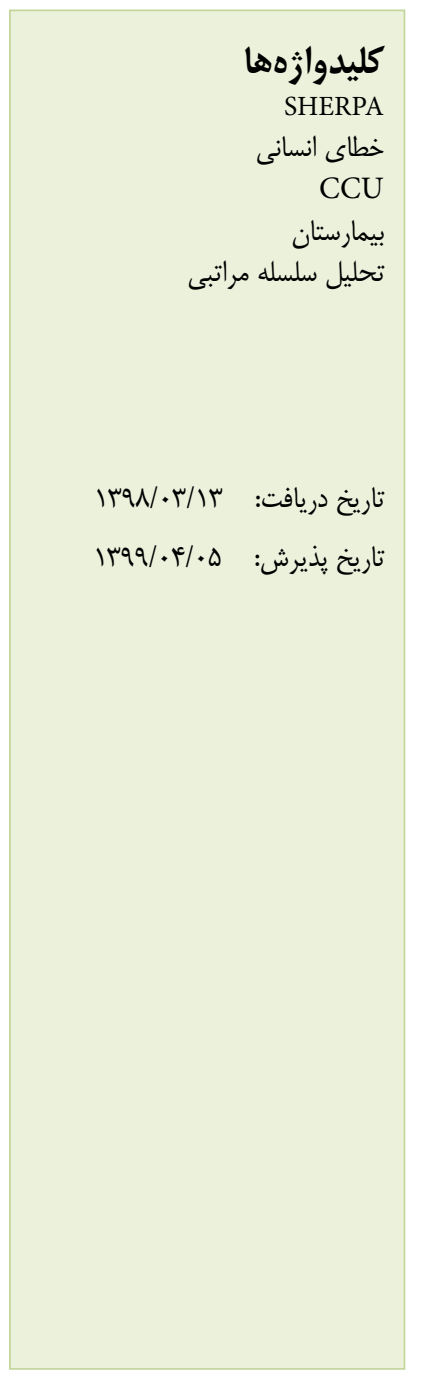

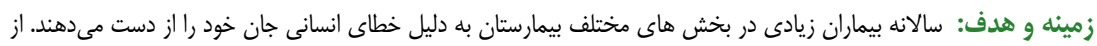

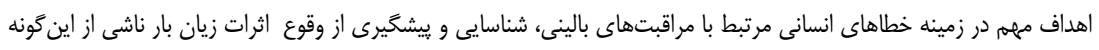

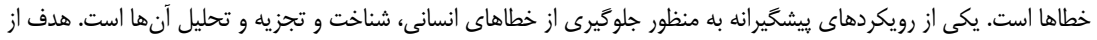

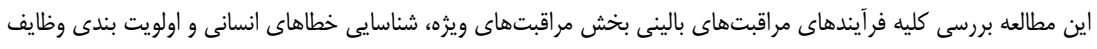
و خطاهاى موجود در اين بخش مى بر باشد.

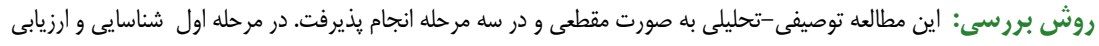

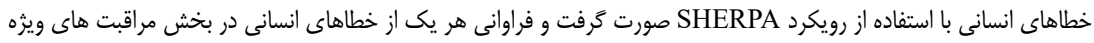

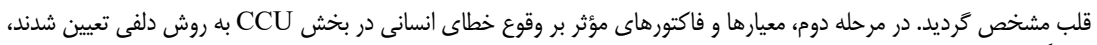

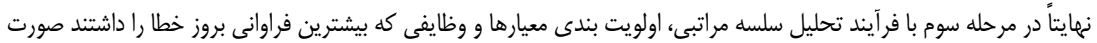

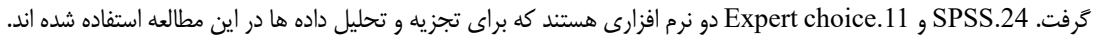

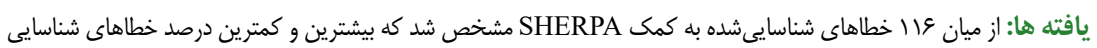

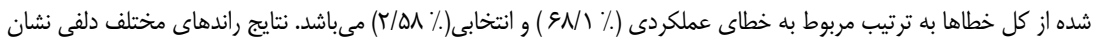

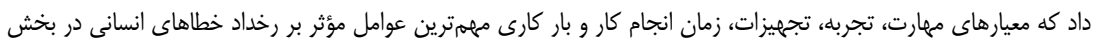

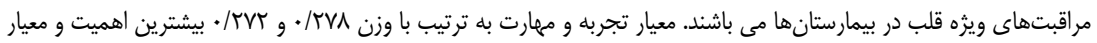

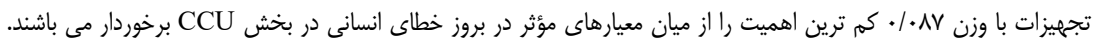

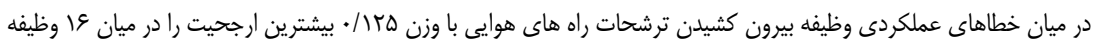

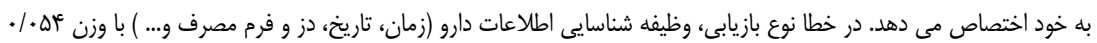

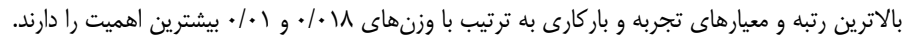

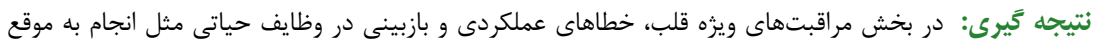

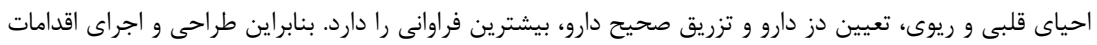

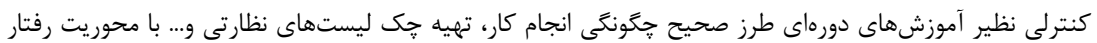

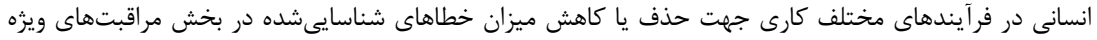

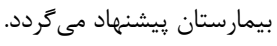
تنعارض منافع: كزارش نشلده /ست. منبع حما يت كنتلده: ندارد.

شيوه استناد به اين مقاله: - مئ Amir Abbasi garmaroudi, Sajjad Mozaffari, Mahshid Bahrami, Hadi Alimoradi Identification. Evaluation and Prioritization of Human Errors in the Nursing Population of the Hospital's Coronary Care Unit. Iran Occupational Health. 2021 (01 June);18:11. 
با مراقبتهاى بالينى، شناسايى و پيشخيرى ازئ وقوع

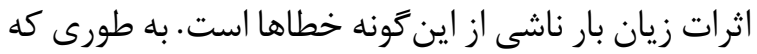

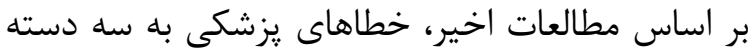

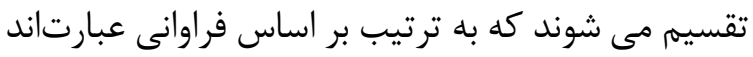

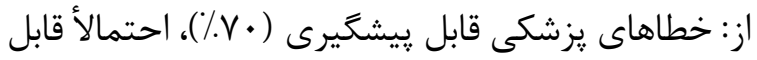

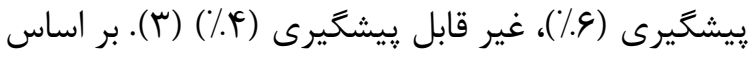

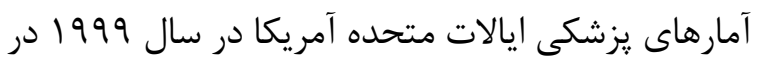

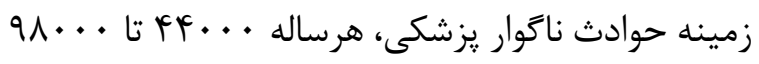

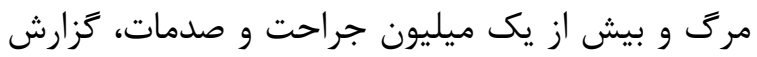

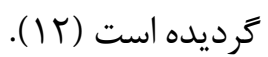

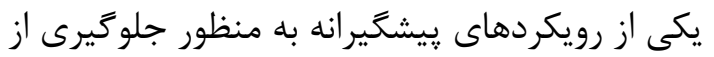

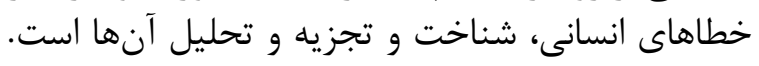

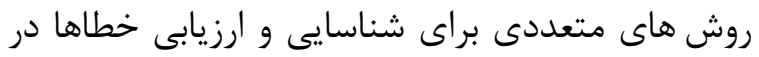

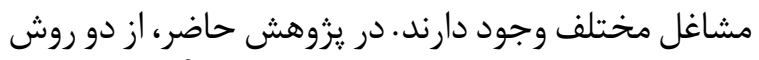

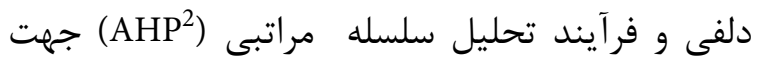

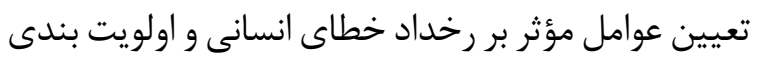

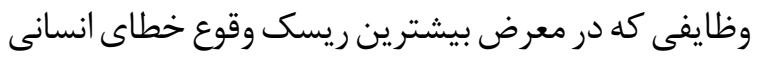

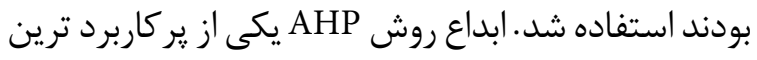

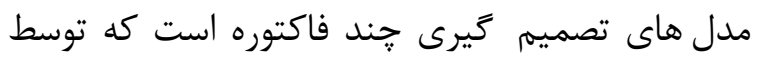

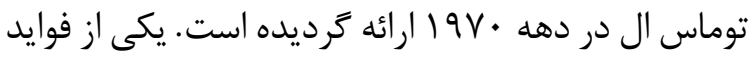

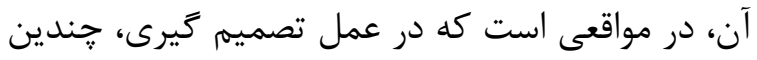
شاخص دخيل هستند. مقايسات زوجى نيز پايه

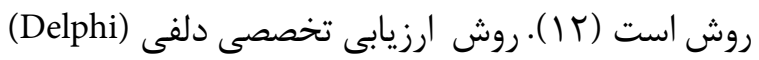

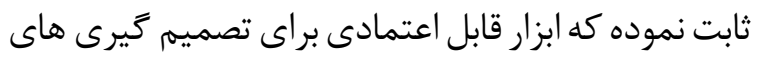

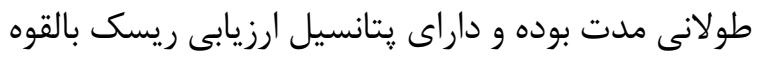

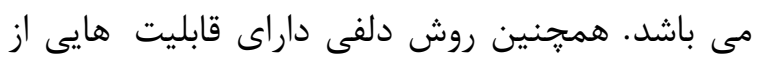

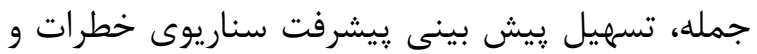

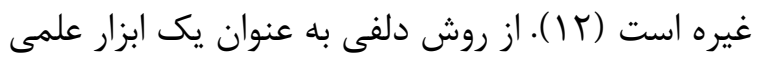

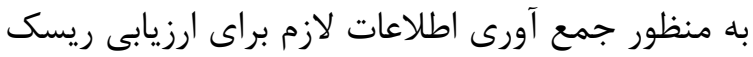

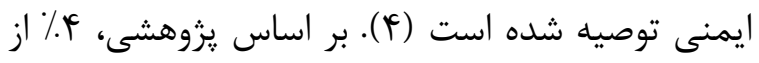

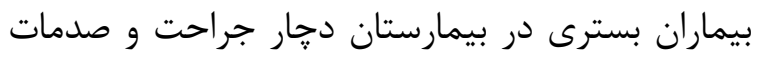

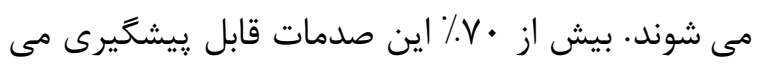

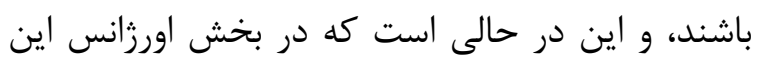

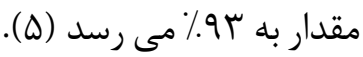

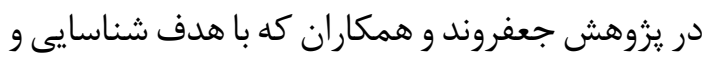

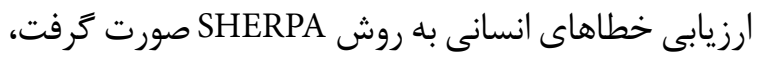

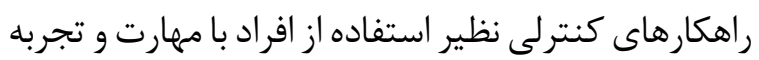

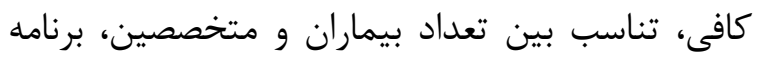

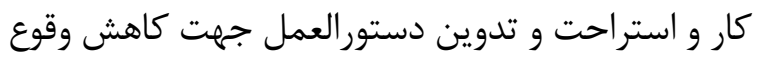

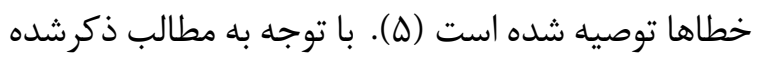
علل شمار زيادى از رخدادهاى بزرى است در سر تاسه به مطاب دنيا اعم

2 Analytical Hierarchy Process

Iran Occupational Health. 2021 (01 June);18: 11
مقال

با وجود يِيشرفت فنّاورى و استفاده از سيستم هاى

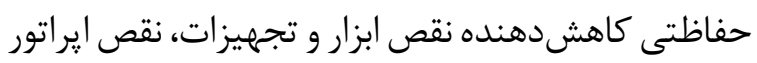

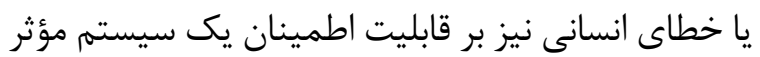

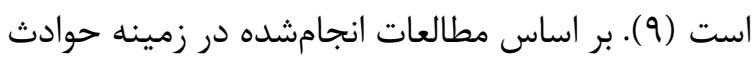

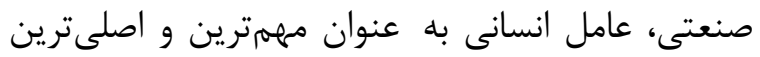

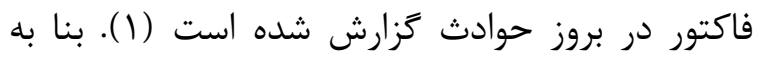

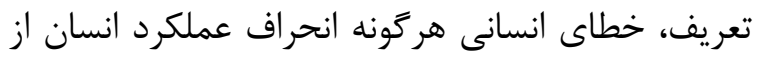

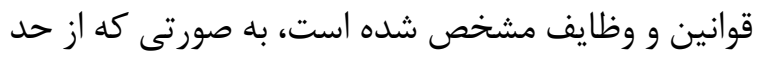

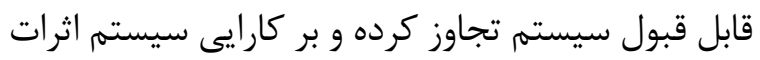

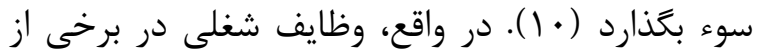

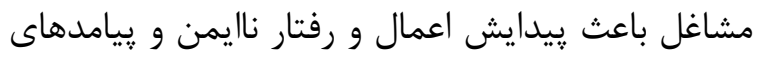

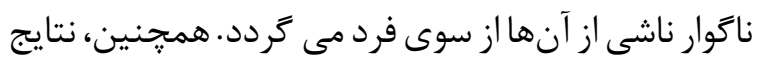

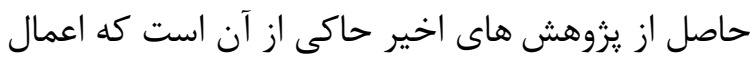

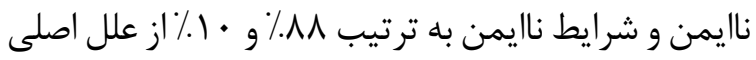

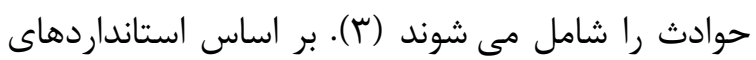

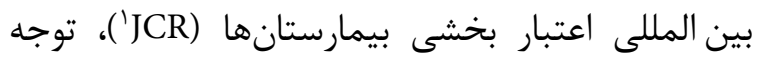

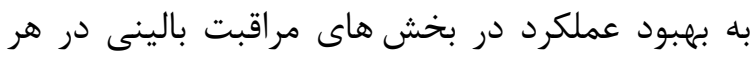

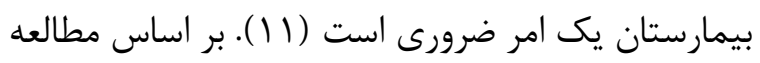

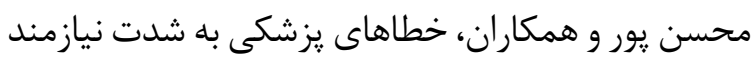

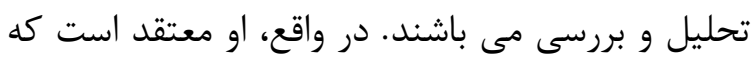

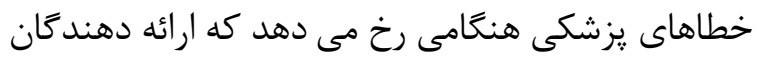

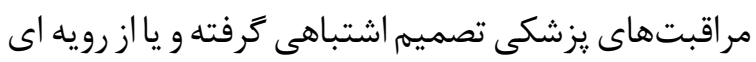

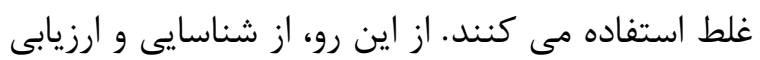

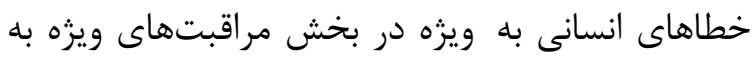

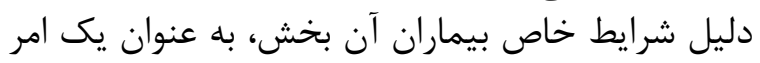

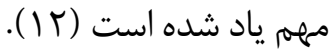

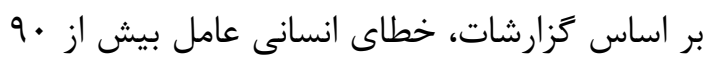

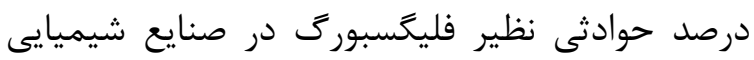

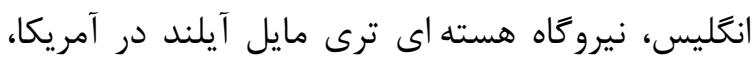

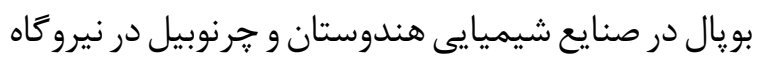

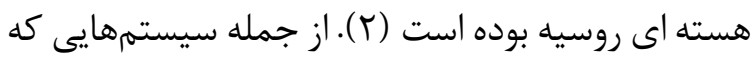

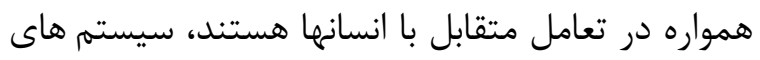

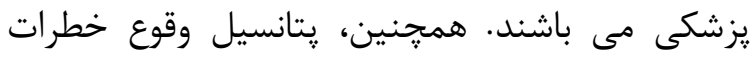

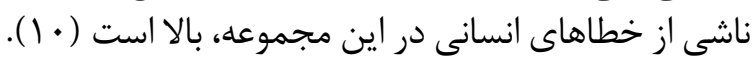

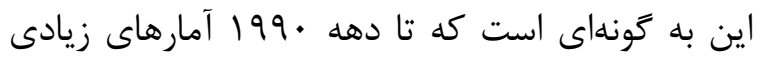

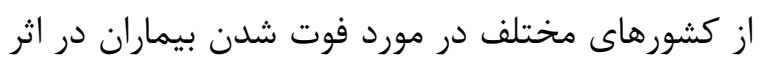

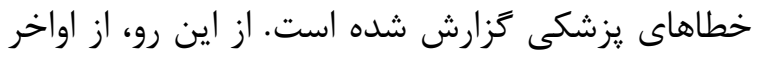

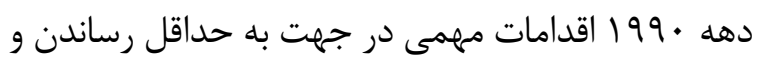

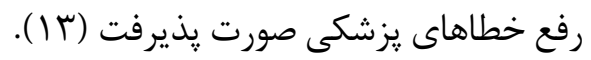
از اهداف مهم در زمينه خطاهاى انسانى مرتبط

1 Joint commission international 
روش تحليل سلسه مراتبى، تمامى وظايفى كه بيشترين

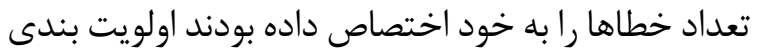

شدند.

در مرحله اول رويكرد سيستماتيك پِيشبينى و

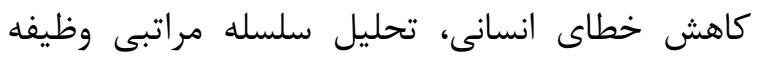

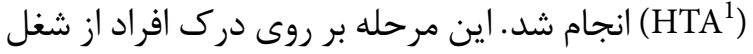

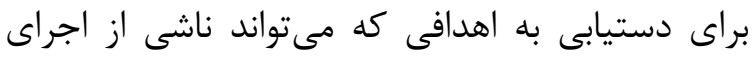
برنامهاى عملياتى و يا دستور العمل ها باشد، تكيه دافيه دارد.

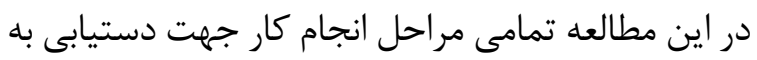

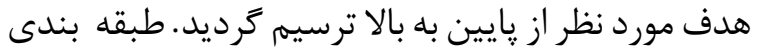

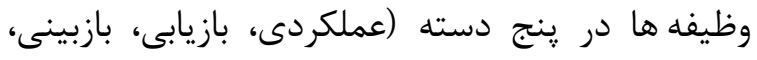

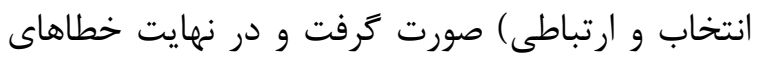
شناسايى شده برحسب وظيفه بر اساس كاربرد

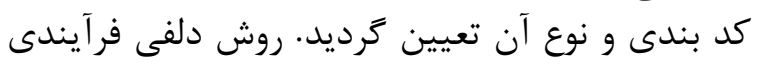

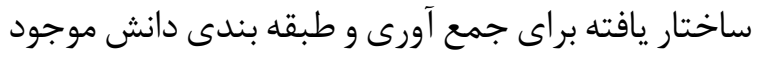

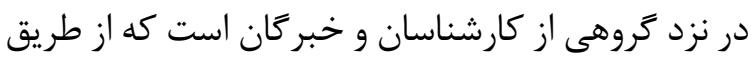

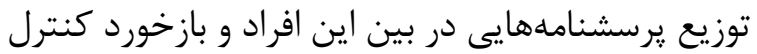

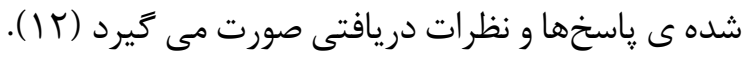

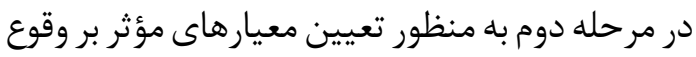
خطاى انسانى در بخش

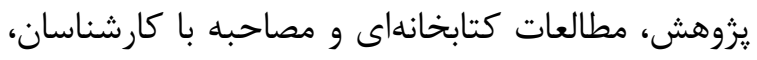

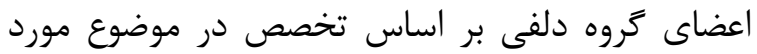

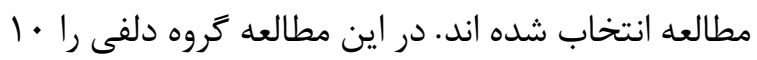
نفر از اساتيد دانشگاه، دانشجويان مقطع دكترى در در رشته دانه

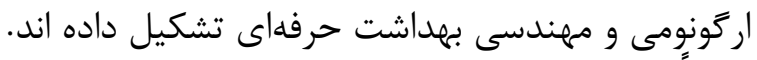

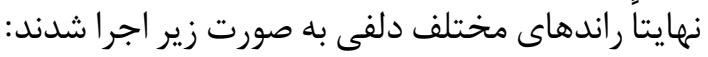

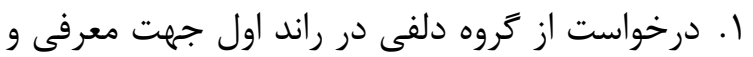
ريشنهاد معيارهاى مرتبط با وقوع درو انواع خطاى انسانى

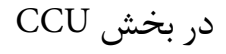

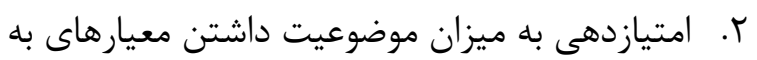

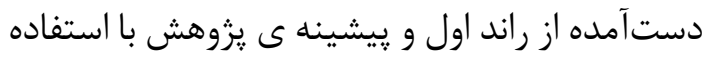

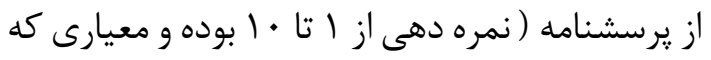

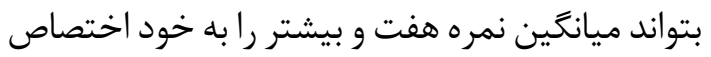

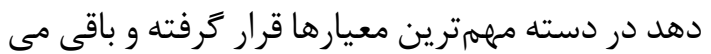

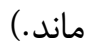
r. حذف معيارهايى كه امتياز لازم را كسب نكرده بودند و

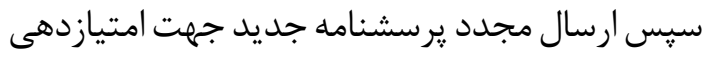
مجدد به معيار هاى باقيمانده

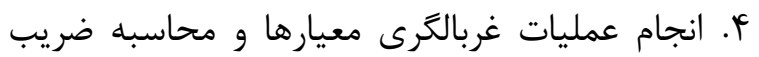

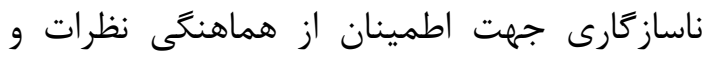
خاتمه دادن به راندهاى دلفى (در غير اين صورت النمات
از صنعتى و يزشكى خطاى انسانى است.كاهش خطاهاى

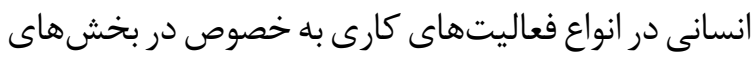

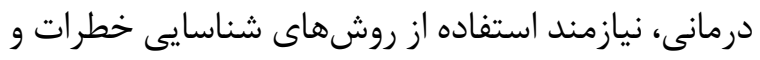

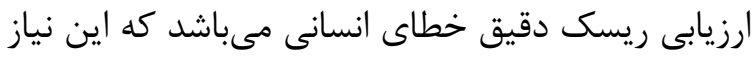

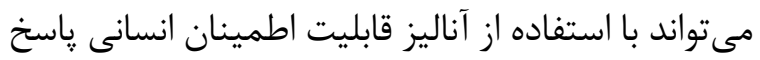

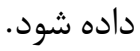

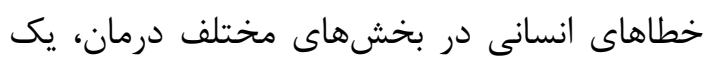

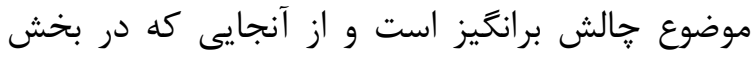

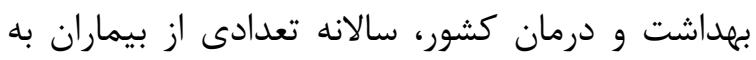

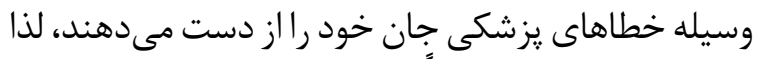

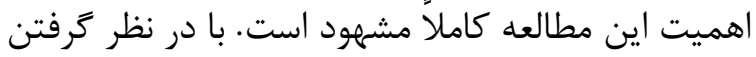
كارايى بالاى روش انسانى در مراكز درمانى، هدف اين مطالعه آن است كه

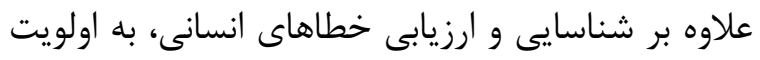

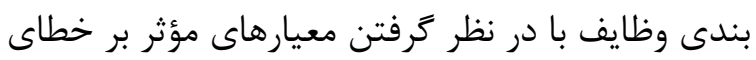

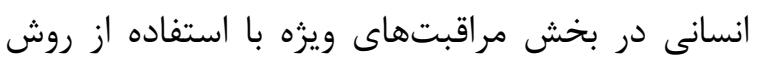

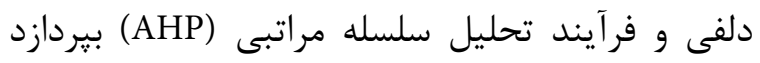

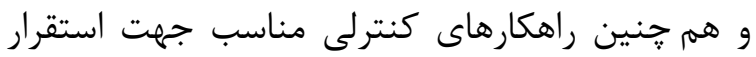

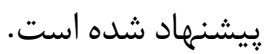

$$
\text { روش بروسى }
$$

اين مطالعه توصيفى-تحليلى به صورت مقطعى براى براى

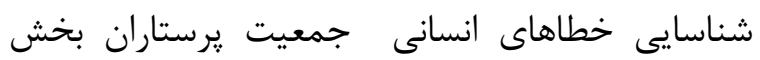

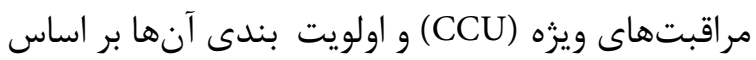

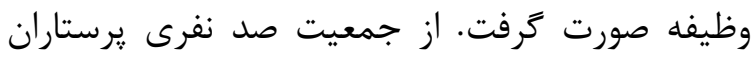

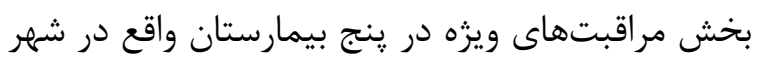

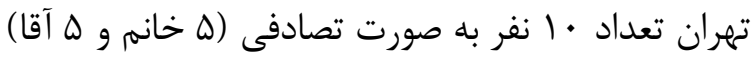

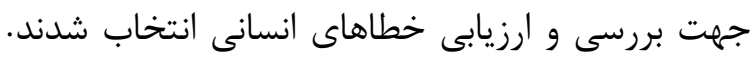

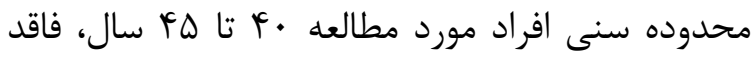

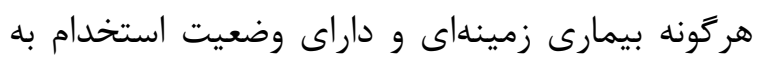

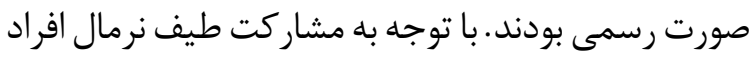

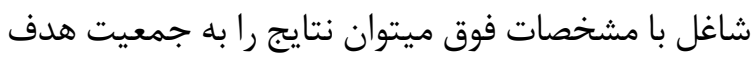
تعميم داد. تكنيك SHERPA مى تواند طيف وسيعى از فرآيندهاى

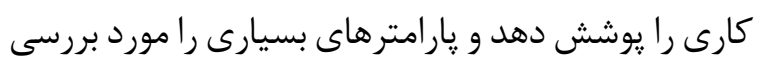

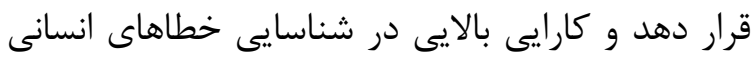

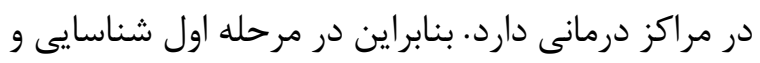

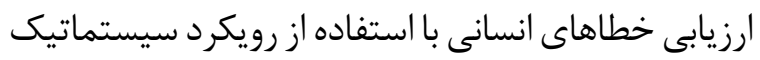

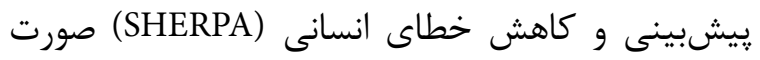

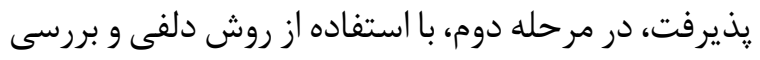

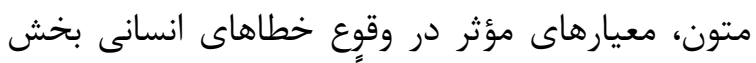

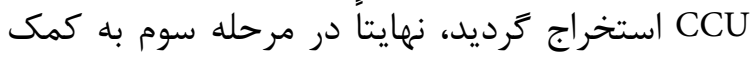


• 1/ • باشد ساز ₹ارى مقايسات قابل قبول بوده و در غير

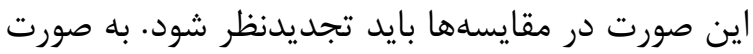

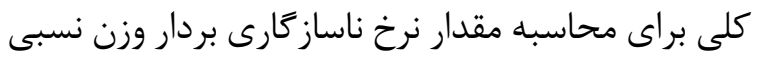

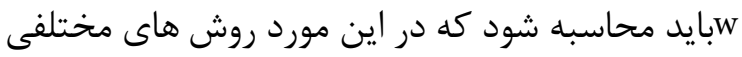

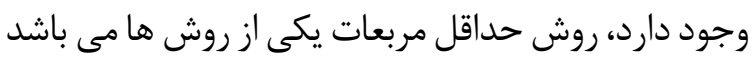

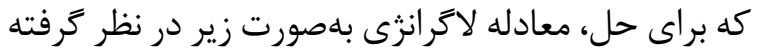

مىشود:

$$
\operatorname{Min} z=\sum_{\mathrm{i}=1}^{\mathrm{n}} \cdot \sum_{j=1}^{\mathrm{n}} \cdot\left(\mathrm{a}_{\mathrm{i}, \mathrm{j}} \mathrm{W}_{\mathrm{j}}-\mathrm{Wi}\right)^{2}
$$

St : $\sum_{i=1}^{n} W i=1$

$\sum_{\mathrm{i}=1}^{\mathrm{n}} \cdot \sum_{j=1}^{n} \cdot\left(\mathrm{a}_{\mathrm{i}, j} \mathrm{~W}_{\mathrm{j}}-\mathrm{Wi}^{2}\right)^{2} \mathrm{~N}\left(\sum_{i=1}^{n} W i-1\right) \mathrm{L}=$

$\sum^{n}{ }_{i=1}\left(a_{\mid l} W_{l}-W_{i}\right) a_{11}-\sum_{j=1}^{n}\left(a_{1 j} W_{j}-W_{1}\right)+\Lambda=0$

شاخص ناسازگارى (I.I) به صورت زير تعريف مىشود:

$$
I . I=\frac{\max -\mathrm{n}}{\mathrm{n}-1}
$$

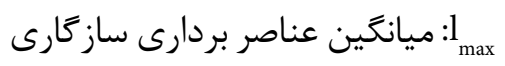

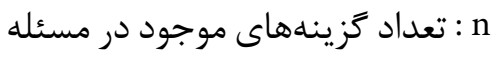

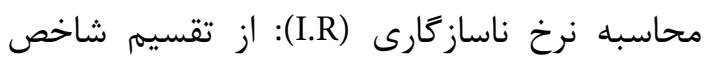

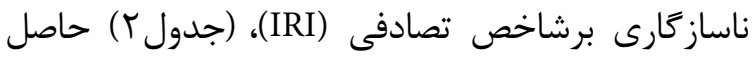

$$
\text { I.R=I.I }
$$

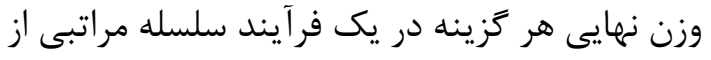

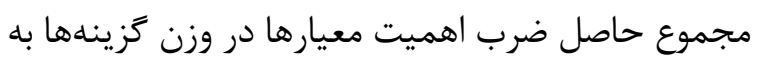

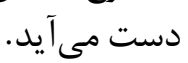

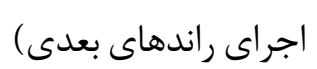

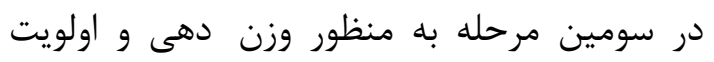

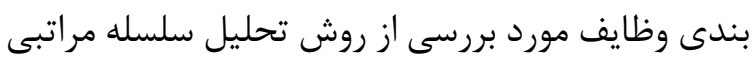

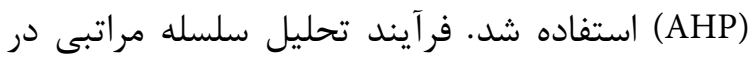

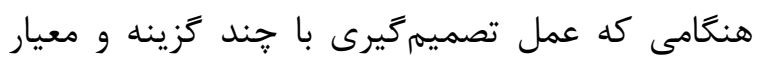

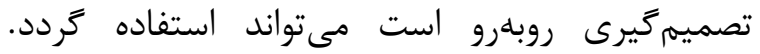

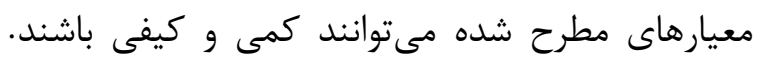

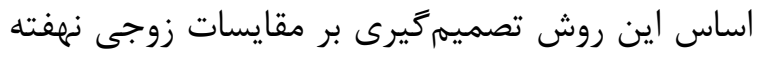

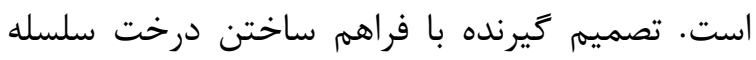

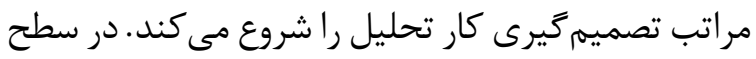

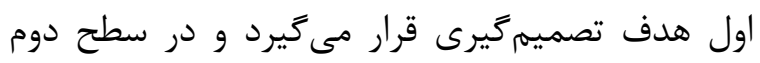

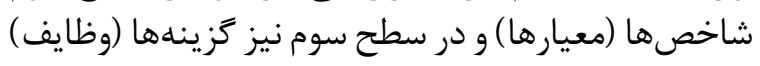

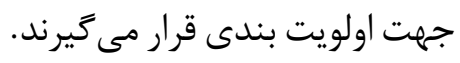

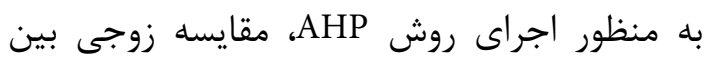

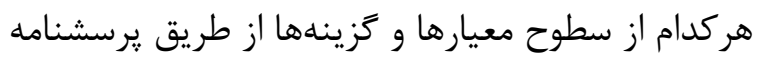

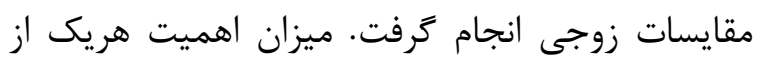

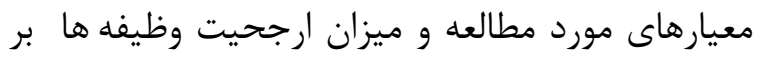

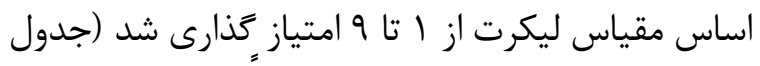

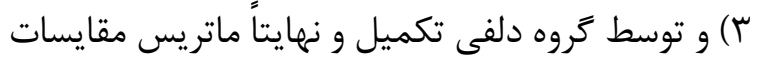

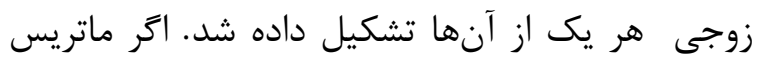

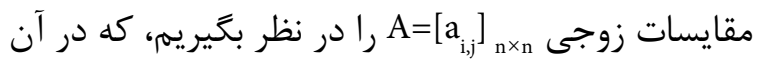

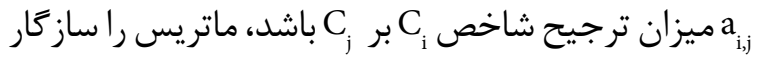

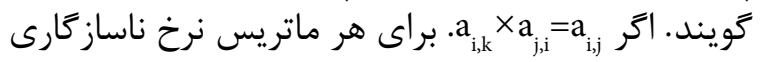

\begin{tabular}{|c|c|c|c|c|}
\hline ارتباطى & بازيابى & انتخابى & بازبينى & عملكردى \\
\hline تجهيزات & انخيزش & مهارت & شرايط كار & ارتباطات ضعيف \\
\hline ارتباطات & آموزش & تجربه & مديريت & مسائل امنيتى \\
\hline خستَى & تجهيزات & آموزش & ميزان حقوق دريافتى & كمبود زمان انجام كار \\
\hline ارتباطات & شرايط & بار كارى & آموزش & خطمشى سازمانى \\
\hline آموزش & تجهيزات & دقت & تجهيزات & تجهيزات \\
\hline تجربه & مهارت & هوشيارى & خطاى سيستم & كاليبره نبودن دستگًاه \\
\hline كار تكرارى & دستور العمل & اطلاعات & يروتكل كارى & شرايط كارى \\
\hline \multirow[t]{3}{*}{ مهارت } & آموزش & شرايط كارى & زمان & آموزش \\
\hline & خستخى & شفاف بودن مسئوليت & دانش & مهارت \\
\hline & باركارى & & مهارت & تجربه \\
\hline
\end{tabular}

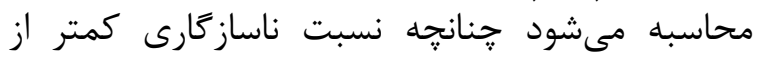

\section{جدول ا. ليست معيارهاى مؤثر بر خطاى انسانى در بخش CCU (استخراج شده از روش دلفى و پيشينه يزوهش)}

\begin{tabular}{|c|c|c|c|c|c|c|c|c|c|}
\hline $\mathrm{N}$ & $r g l$ & $r$ & f & $\Delta$ & 4 & V & $\wedge$ & 9 & 1. \\
\hline RI & . & $\cdot \mid \Delta \Lambda$ & $\cdot / 9$ & $1 / 1 T$ & $1 / T F$ & $1 / T T$ & $|/ 4|$ & $1 / 4 \Delta$ & $1 / \Delta 1$ \\
\hline
\end{tabular}

جدول r. (1) شاخص تصادفى 
شناسايى، ارزيابى و اولويتبندى خطاهاى انسانى در جمعيت يرستاران

\begin{tabular}{|c|c|c|}
\hline \multicolumn{3}{|c|}{ جدول ب. ارزش كذارى شاخصها نسبت به هم } \\
\hline توضيح & 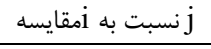 & 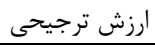 \\
\hline اهميت برابر دارندز نسبت به أزَزينه يا شاخص & اهميت برابر & 1 \\
\hline كمى مهمتر است.j نسبت به أكزينه يا شاخص & نسبتاً مهمتر & r \\
\hline مههمتر است.j نسبت به iٔزَ ينه يا شاخص & مهمتر & $\Delta$ \\
\hline است.j داراى ارجحيت خيلى بيشترى از iَز ينه يا شاخص & 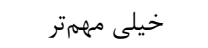 & $\checkmark$ \\
\hline نيست.j مهمهر و قابلمقايسه با j از iكزينه يا شاخص مطلقاً & كاملاً مهم & 9 \\
\hline ارزشهاى ميانى بين ارزشهاى ترجيحى را نشان مى دهد مثلاً ^، بيانكر اهميتى زيادتر از V و & -- & r r r \\
\hline
\end{tabular}

در كل 1 الخطاى احتمالى شناسايى شد (جدول \&). كه يافته ها

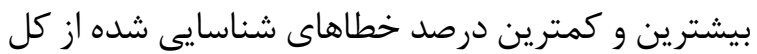

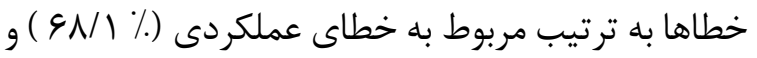

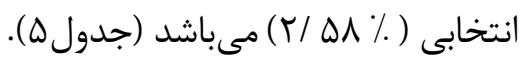
نتايج راندهاى مختلف دلفى نشان داد إد معيارهاى

نتايج به دست آمده از شناسايى و ارزيابى خطاى

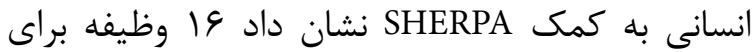

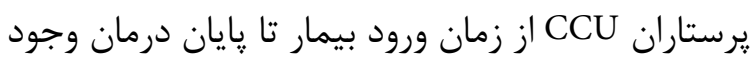
دارد كه بيشترين تعداد خطا ها را به خون خود اختصاص دان دادند.

جدول F. نمونه تكميلشده كار برك SHERPA

\begin{tabular}{|c|c|c|c|}
\hline 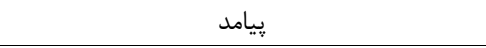 & توصيف خطا & نوع خطا & وظيفه شغلى \\
\hline اشتباه در انتخاب دارو & اشتباه در خواندن نام دارو & $\mathrm{R} 2$ & 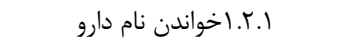 \\
\hline ممكن است سرنگ يا تجهيزات اشتباه انتخاب شود & عدم توانايى در كار كذاشتن يمب تزريق & A9 & F \\
\hline 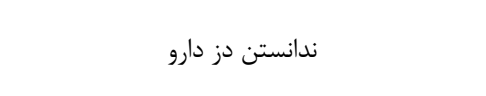 & دارن اطلاع بهات غلط در خصوص مكان اسمى بيمان & $\mathrm{R} 2$ & F. ا. بررسى خارت بيماران \\
\hline
\end{tabular}

جدول ه. فراوانى و درصد انواع خطا

\begin{tabular}{|c|c|c|c|c|c|c|c|}
\hline جمع & ارتباطى & & انتخا & & 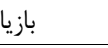 & بازبينى & عملكردى \\
\hline تعداد 1119 & تعداد ه ه & $r$ & ت تعداد & $r \cdot$ & تعداد & 9 & Vع تعداد V9 \\
\hline درصد \% . 1. & درصد \% آر & $r / \Delta \Lambda$ & درصد٪ & $I V / T F$ & درصد \% & درصد \% \% & $9 N / 1 \%$ \\
\hline
\end{tabular}

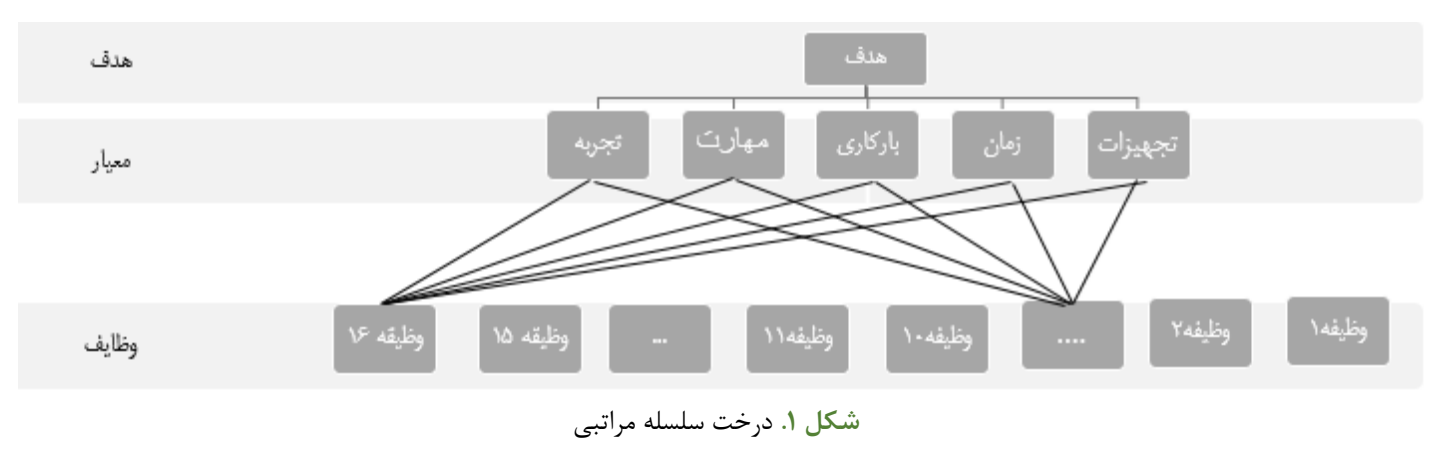

جدول 9. ميزان وزن (اهميت) معيارها

\begin{tabular}{|c|c|c|c|c|c|}
\hline زمان & تجهيزات & تجربه & بار كارى & مهارت & معيار \\
\hline.$/ 190$ & $\cdot / \cdot \wedge \vee$ & $\cdot / T V \Lambda$ & .1191 &.$/ T V Y$ & وزن \\
\hline
\end{tabular}


جدول V. رتبهبندى \& وظيفهى داراى بيشترين وزن به تفكيك معيارها

\begin{tabular}{|c|c|c|c|c|c|c|c|c|c|c|}
\hline وزن & مهارت & وزن & باركارى & وزن & تجربه & وزن & تجهيزات & وزن & زمان & \\
\hline$\cdot / \cdot r \Delta$ & ترشحات راههاى &.$|\cdot \mu|$ & $\begin{array}{c}\text { انجام اقدامات اوليه } \\
\text { CPR }\end{array}$ & .1 .49 & اولجام اقدامات &.$/ \cdot 14$ & گِمٍ تزريق & $\cdot|\cdot r|$ & ترشحات راههاى كشيدن & وظيفها \\
\hline . $/ \cdot T V$ & موارد اورخانسى لولى دَارى در & . & ترشحات راههاى كشيدن & $\cdot / \cdot r \Delta$ & بيرون كشيدن & .1 .14 & 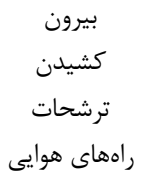 & .1 .11 & $\begin{array}{c}\text { انجام اقدامات اوليه } \\
\text { CPR }\end{array}$ & وظيفه \\
\hline & عضلانى و وريدى تزريق & $.1 \cdot 11$ & موارد اورزانسى لوله گذارى در &.$/ \cdot r V$ & يٍَّ تزريق & $.1 \cdot 11$ & لولهَّام & .1 .18 & $\begin{array}{l}\text { اقدامات فورى براى } \\
\text { dysrhythmia }\end{array}$ & وظيفه \\
\hline . & $\begin{array}{c}\text { انجام اقدامات اوليه } \\
\text { CPR }\end{array}$ & $.1 .1 \mathrm{~V}$ & $\begin{array}{c}\text { اقدامات فورى براى } \\
\text { dysrhythmia }\end{array}$ & $\cdot 1 \cdot r_{\Delta}$ & لوارو لوذارى در اورثام & & & r.|. & عضلانى و وريدى انجام تزريق & وظيفه F \\
\hline
\end{tabular}

جدول ^. وزن (ارجحيت) وظيفهها به تفكيك معيارها

\begin{tabular}{|c|c|c|c|c|c|c|c|c|c|}
\hline 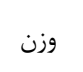 & معيار شاخص & وزن نسبت به هدف & زمان & تجهيزات & تجربه & بار كارى & مهارت & وظيفه & خنطا \\
\hline .1 .49 & تجربه &.$/ N F$ & .1 .11 & 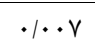 & .1 .49 & $\cdot|\cdot r|$ &.$/ \cdot r$ & انجام اقدامات اوليه CPR & \\
\hline.$/ \cdot \mathrm{TV}$ & 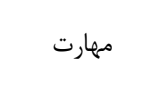 & .1 .91 & .1 .19 & $.1 \cdot 11$ & $.1 \cdot r_{\Delta}$ & $\cdot 1 \cdot 11$ &.$/ \cdot \mathrm{TV}$ & اورزام لوله كذارى در موارد & \\
\hline.$/ \cdot T Y$ & 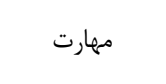 & $\cdot / \cdot \wedge \Delta$ & .1 .18 & $\cdot 1 \cdot 1$ & .1 .19 & $.1 .1 \mathrm{~V}$ & $.1 \cdot T r$ & $\begin{array}{r}\text { اقدامات فورى براى } \\
\text { dysrhythmia }\end{array}$ & \\
\hline $.1 \cdot 19$ & تجربه & $\cdot / \cdot \Delta \varphi$ &.$/ \cdot 1$ & $\cdot 1 \cdot \Delta$ & .1 .19 &.$/ .14$ & $.1 \cdot 14$ & ثبكيار جه دادها ديمارستان سيستم & 豙 \\
\hline$\cdot / \cdot r \Delta$ & مهارت و تجربه &.$/ 1 T \Delta$ & $\cdot|\cdot r|$ & .1 .14 & . $/ \cdot \mu_{\Delta}$ & . I. Tr &.$/ \cdot \mu_{\Delta}$ & 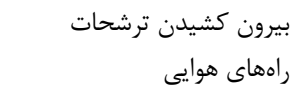 & $\hat{y}$ \\
\hline r & 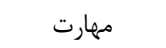 & $\cdot / \cdot \vee$ & . & $\cdot 1 \cdot \Delta$ & .1 .19 &.$/ \cdot 14$ & 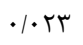 & انجام تزريق عضلانى و وريدى & \\
\hline $.1 \cdot 14$ & بار كارى & $\cdot \mid \cdot \Delta 1$ & .1 .99 & 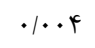 & $\cdot / \cdot 1$ & $\cdot 1 \cdot 1 f$ & $\cdot 1 \cdot 1$ & متصل كردن ست سرم & \\
\hline.$/ \cdot t V$ & تجربه & .1 .9 & .1 .14 &.$/ \cdot 14$ &.$/ \cdot T V$ &.$/ \cdot 1 \mathrm{~V}$ & $.1 \cdot 11$ & كاركذارى يمب تزريق & \\
\hline$\cdot / \cdot 11$ & تجربه & $\cdot / \cdot \Delta r$ & $.1 \cdot 9$ & $\cdot / \cdot r$ & $.1 \cdot 11$ & $.1 \cdot 1$ & .1 .99 & ثبت اطلاعات دارو & \\
\hline .1 .99 & تجربه & $.1 \cdot r \mu$ & $.1 \cdot .4$ & . & .1 .99 & $\cdot / \cdot 1$ & $\cdot / \cdot 1$ & شناسايى بيمار & קִ \\
\hline.$/ \cdot 1 f$ & 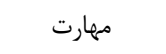 &.$/ .49$ & $.1 . .4$ & $\cdot / \cdot r$ & $\cdot 1 \cdot .9$ & $\cdot / \cdot v$ & $.1 \cdot 14$ & يايش قلب & \\
\hline .1 .99 & تجربه & $.1 \cdot \mu r$ & $.1 \cdot 9$ & . & .1 .99 & $\cdot / \cdot \wedge$ & $\cdot / \cdot 1$ & شناسايى بيمار & \\
\hline $.1 \cdot 10$ & تجربه و مهارت & .1 .49 & $\cdot 1 \cdot v$ & $/ \cdot r$ & . & $\cdot 1 \cdot .4$ &.$/ \cdot 1 \mathrm{~V}$ & خواندن دز دارو & $\vec{?}$ \\
\hline.$/ \cdot 1 \mathrm{~V}$ & تجربه و مهارت & $\cdot 1 \cdot \Delta F$ & $\cdot / \cdot V$ & 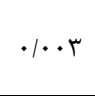 &.$/ .14$ &.$/ .1$ & . $/$ rt & شاريخ، فرم مصرف و.... ) & \\
\hline$\cdot / \cdot v$ & مهارت و تجربه &.$/ \cdot T V$ & 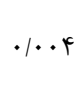 & $\% r$ & $\cdot \cdot \cdot v$ & $.1 \cdot 4$ & $\cdot / \cdot v$ & شوى DC ،نتخيم و بهكار كيرى & 立 \\
\hline .1 .99 & 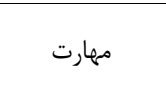 &.$/ \cdot \mathrm{TV}$ & $\% f$ & $\% r$ & $\cdot \cdot \cdot v$ & $\cdot / \cdot \Delta$ & .1 .99 & آموزش قبل، حين و بعد از & $\overline{3}$ \\
\hline .1 .99 & تجربه & $\cdot|\cdot r|$ & $\% q$ & $\%$ r & $.1 . .4$ & $\cdot / \cdot r$ & $\cdot 1 \cdot \Delta$ & اطليه احياى از كلب شروع مراحل عروق & th \\
\hline
\end{tabular}


شناسايى، ارزيابى و اولويتبندى خطاهاى انسانى در جمعيت يرستاران

جدول 9. ميانگَين وزن خطاها

\begin{tabular}{|c|c|c|c|c|c|}
\hline ارتباطى & انتخابى & بازيابى & بازبينى & عملكردى & نوع خطا \\
\hline $.1 \cdot 4 \lambda$ &.$/ \cdot T V$ &.$/ I M F$ & $\cdot 1 \cdot \mathrm{Vr}$ & $\cdot / V \Delta T$ & وزن ميانكَين \\
\hline
\end{tabular}

از رويكرد سيستماتيك يُشبينى و كاهش خطاى انسانى

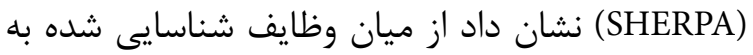
وسيله تحليل سلسله مراتبى وظيفه (HTA) (HTH)، 19 وظيفان وظيفه

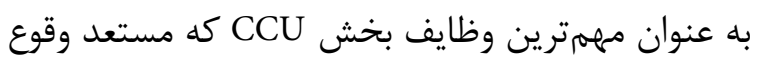

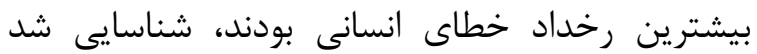

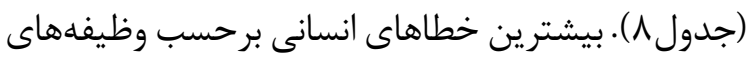
شناسايىشده، از نوع خطاى عملكردى (

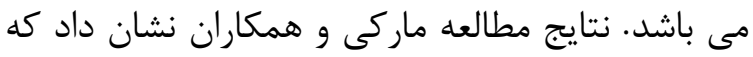

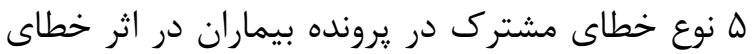

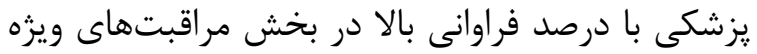

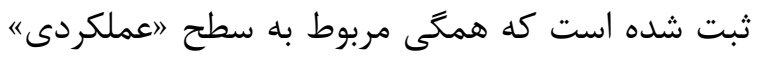
و يا به عبارتى تحليل عقلانى موقعيتهاى كاى كارى بوده

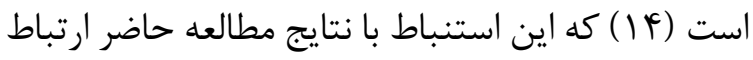
و همخوانى دارد. هم:جنين مطالعه رولستون و همكاران

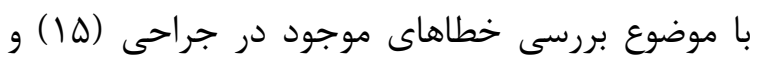

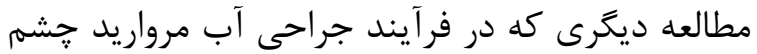

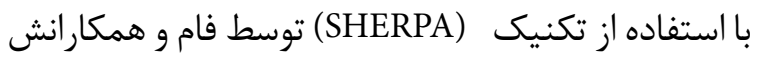

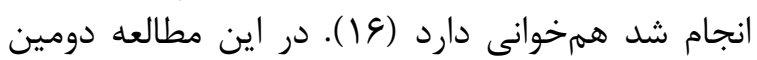

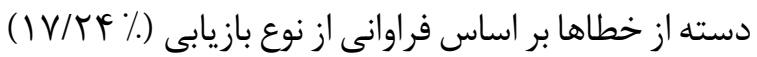

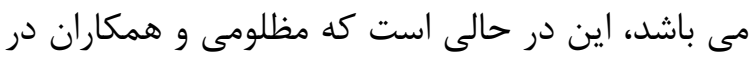

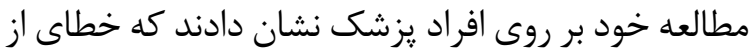

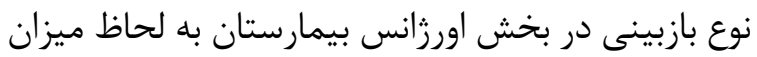

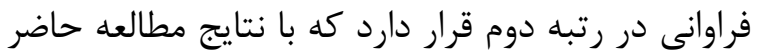

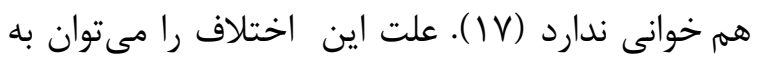

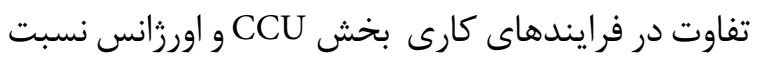

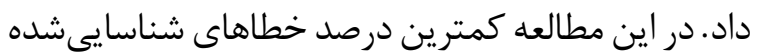

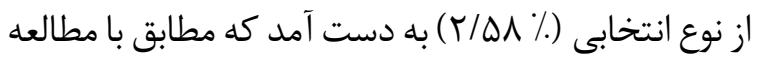

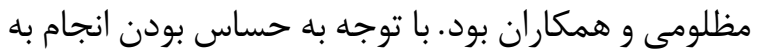

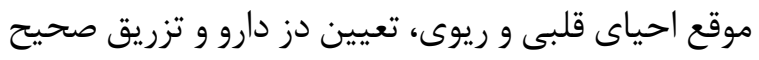
دارو، وظايف از نوع عملكردى و بازيابى بيشي نائرين

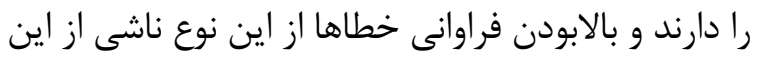

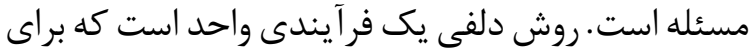

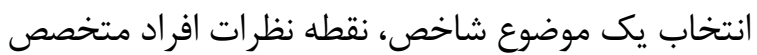

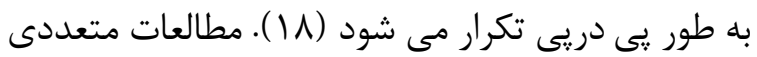

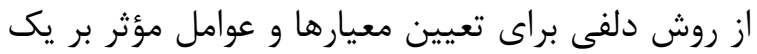

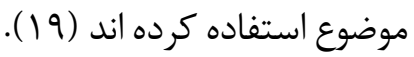
شناسايى مهمهترين معيارها در اسنفاد رخداد خطاهاى
مهارت، تجربه، تجهيزات، زمان انجام كار و باركارى

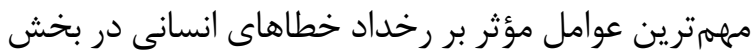

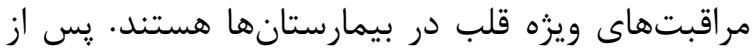

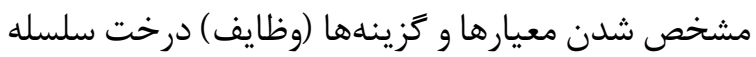

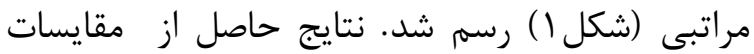

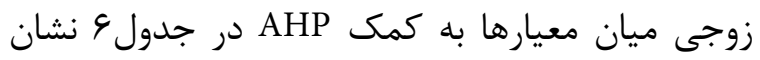

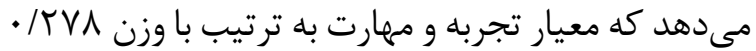

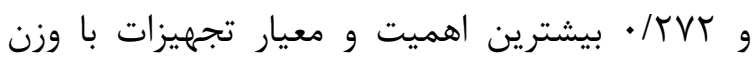

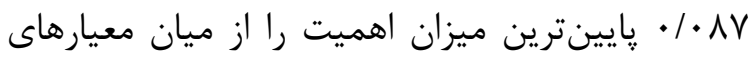

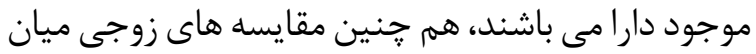

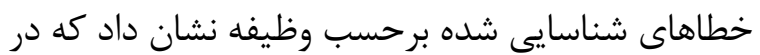

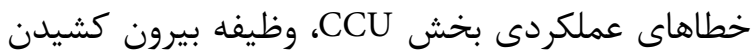

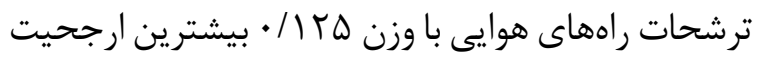

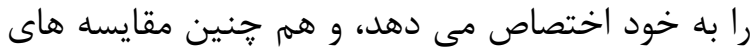

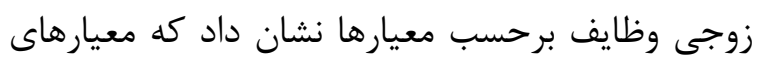

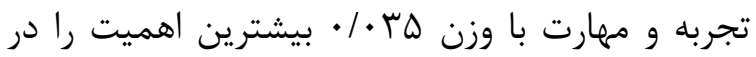

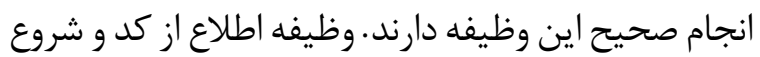

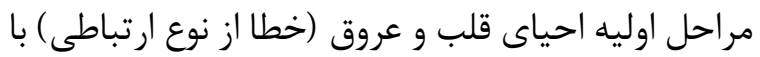

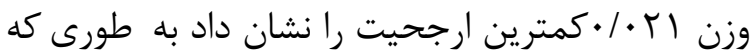

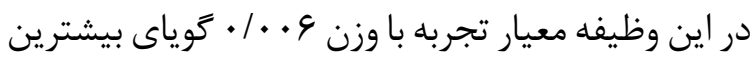

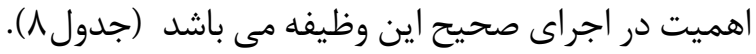

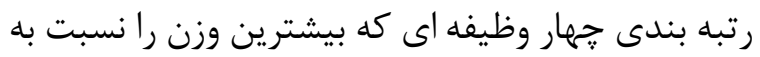
سايرين اخذ كردند به تفكيك معيارها در جدول لون آمده

به عنوان مثال در معيار تجهيزات، وظيفه كار حذارى

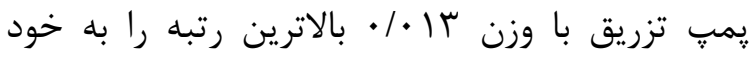

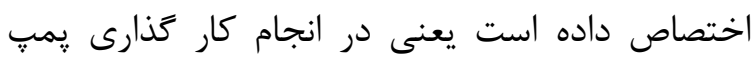

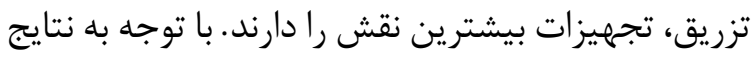

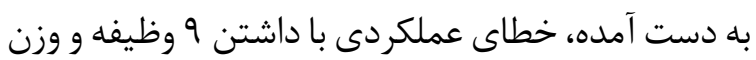

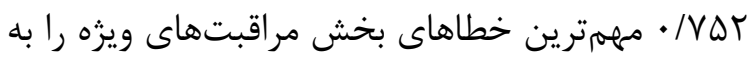

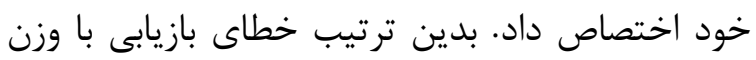

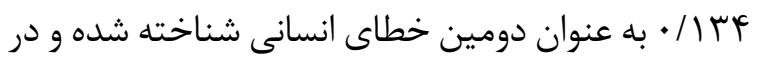

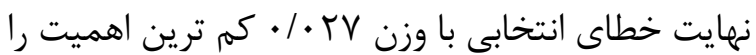

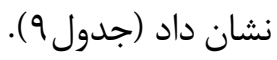

بحث نتايج شناسايى و ارزيابى خطاهاى انسانى با استفاده 
از وظايف تكرارى يرستاران در هر نوبت كارى مى باشد

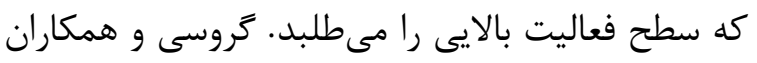

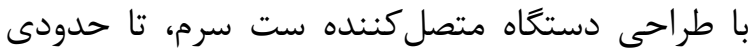

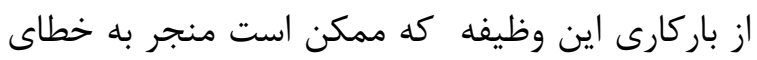

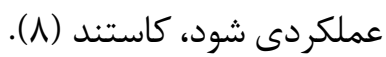

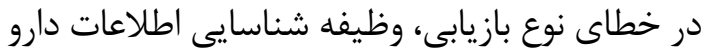

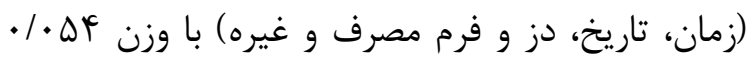

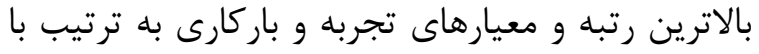

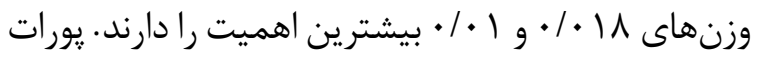

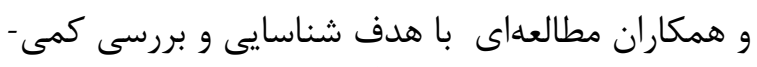

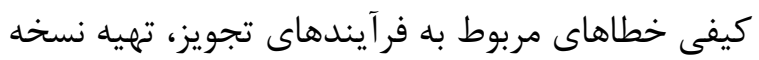

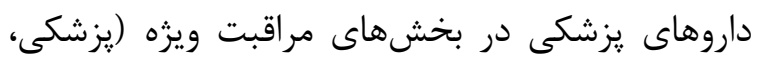

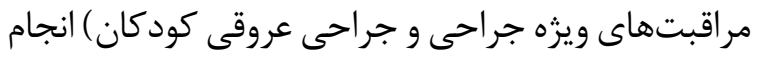
دادند، آنها كزارش كردزئن

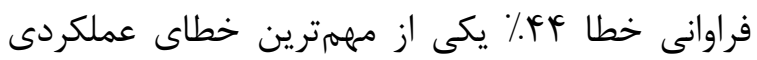

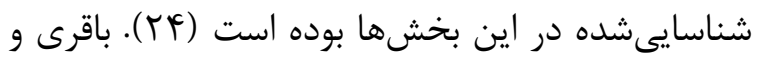

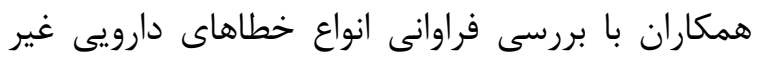

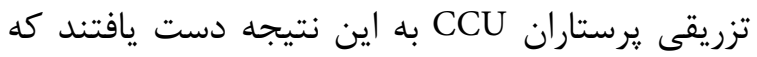

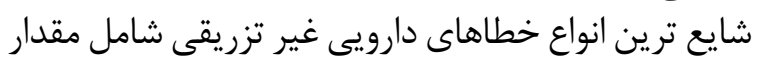

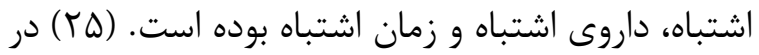

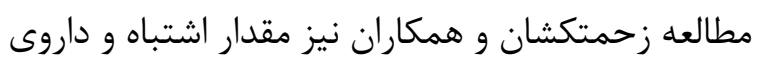

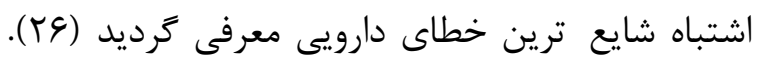

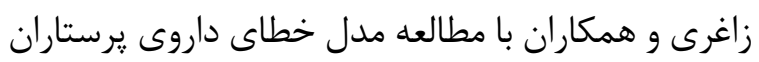

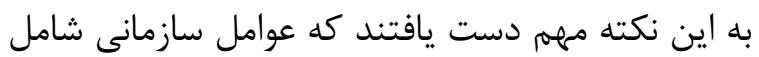

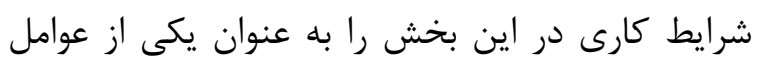

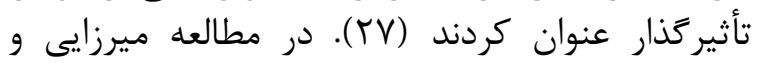

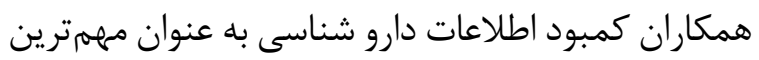

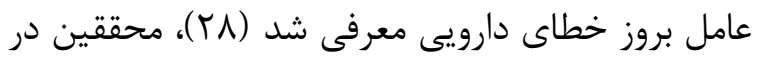

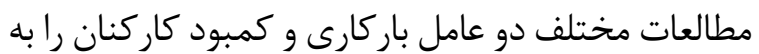

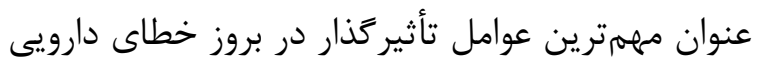

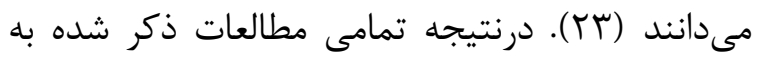

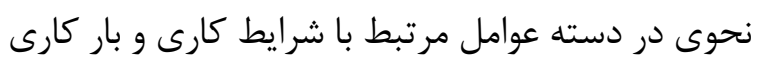
مىباشند كه منطبق با نتايج اين مطالعه است.

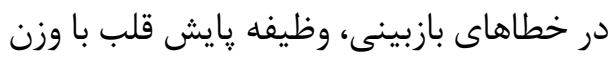

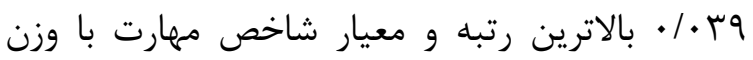

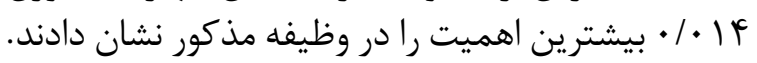

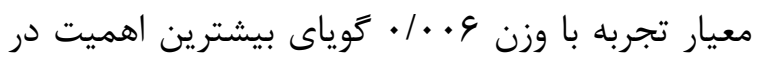

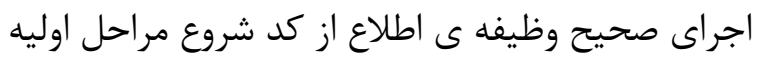

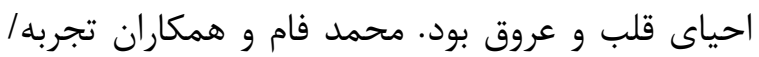

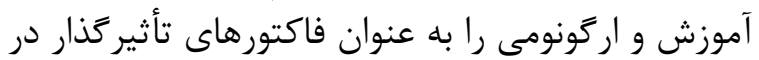

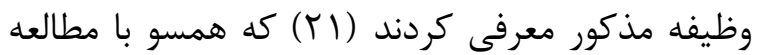

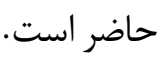

انسانى توسط كروه دلفى بررسى و مشخص شد كه ينج

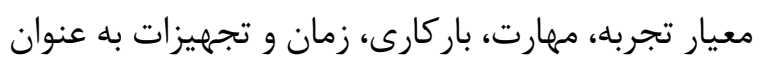

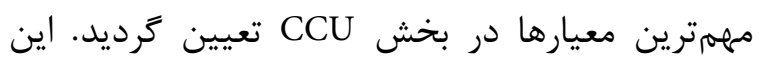

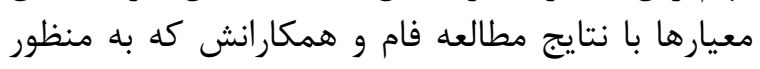

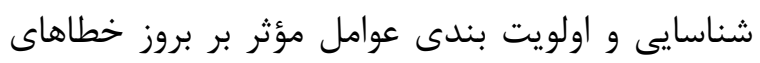

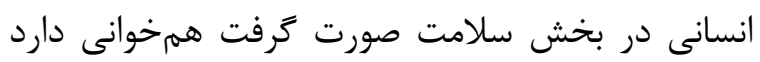

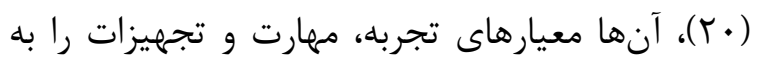

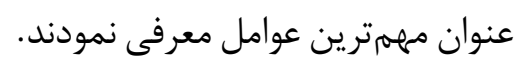

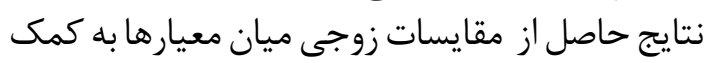

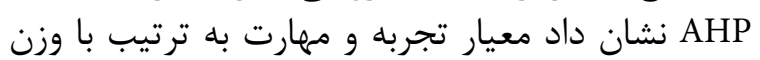
دادن / IVA

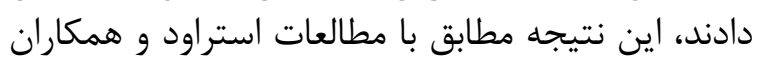

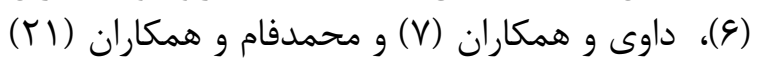

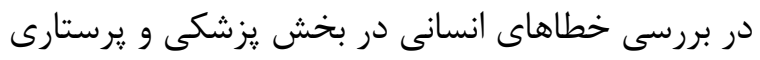

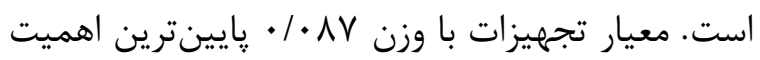

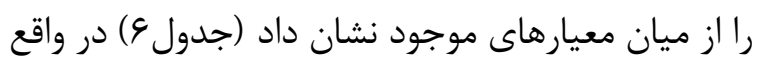

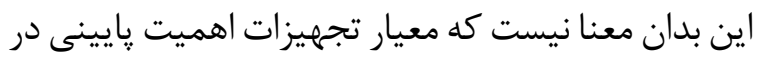

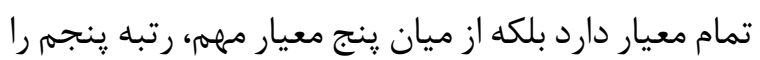
به خود اختصاص داده است.

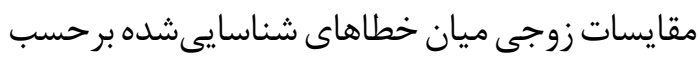

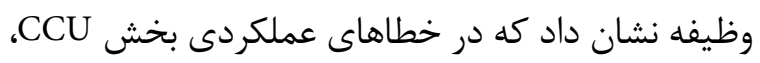

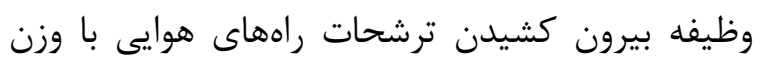

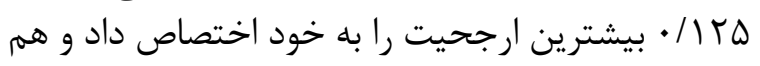

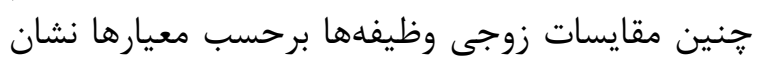

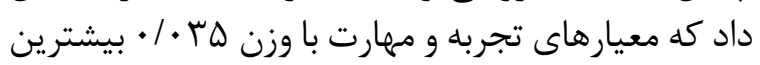

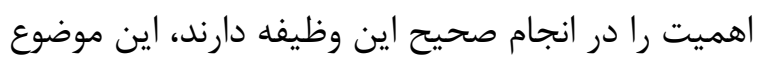

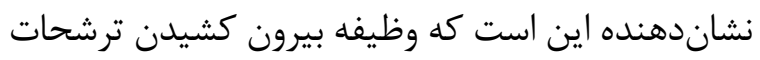

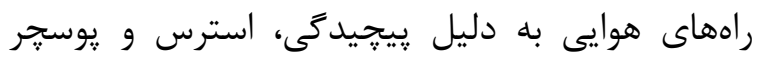

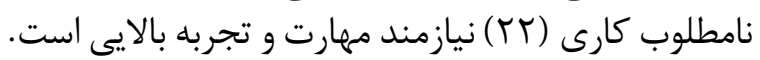

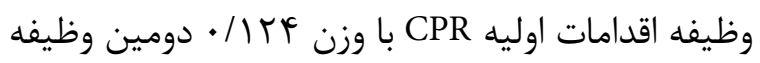

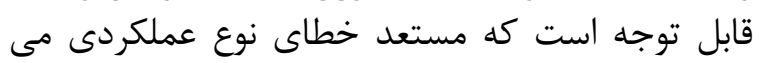

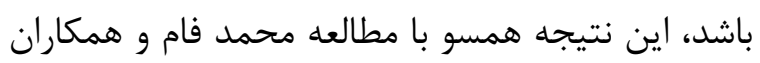

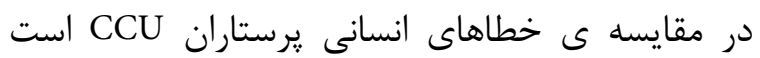

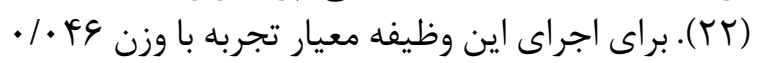

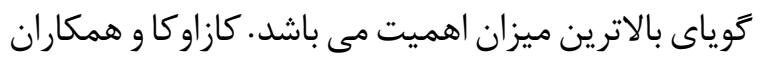

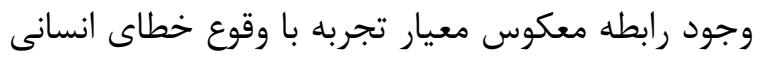

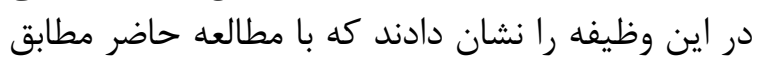

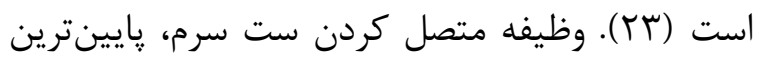

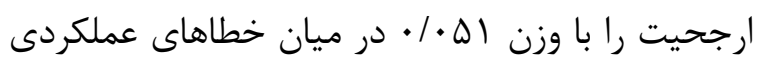

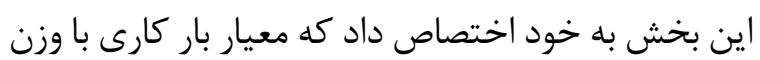

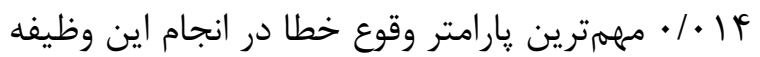

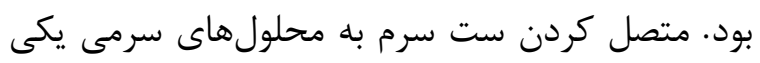


شناسايى، ارزيابى و اولويتبندى خطاهاى انسانى در جمعيت برستاران

$$
\text { تشكر و قدردانى }
$$

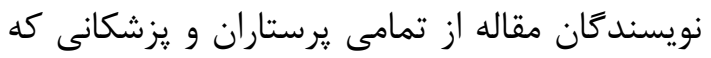

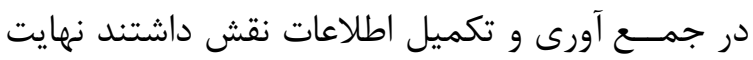

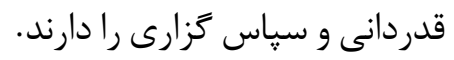

\section{منابع}

1. Mortazavi SB, Mahdavi S, Asilian H, Arghami S, Gholamnia R. Identification and assessment of human errors in srp unit of control room of tehran oil refinery using heist technique. 2008;12(3):308-322. [Persian]

2. Tanha F, Mazloumi A, Faraji V, Kazemi Z, Shoghi M. Evaluation of human errors using standardized plant analysis risk human reliability analysis technique among delivery emergency nurses in a hospital affiliated to Tehran University of Medical Sciences. Journal of Hospital. 2015;14(3):57-66. [Persian]

3. Dastaran S, Hasheinejhad N, Shahravan A, Baneshi M, Faghihi A. Identification and Assessment of Human Errors in Postgraduate Endodontic Students of Kerman University of Medical Sciences by Using the SHERPA Method. Journal of Occupational Hygiene Engineering. 2016;2(4):44-51. [Persian]

4. Markmann C, Darkow I-L, von der Gracht H. A Delphi-based risk analysis-Identifying and assessing future challenges for supply chain security in a multistakeholder environment. Technological Forecasting and Social Change. 2013;80(9):1815-1833.

5. Jafarvand M, Khoshnvaz H, Kazemi S, Varmazyar S, Ghorbanideh M. Identification and Assessment of Human Errors Using SHERPA in the Endodontic Department of Clinic of Dentistry Faculty, Qazvin University of Medical Sciences. Health-Based Research. 2017;3(3):267-276. [Persian]

6. Stroud L, Wong BM, Hollenberg E, Levinson W. Teaching medical error disclosure to physicians-in-training: a scoping review. Academic Medicine. 2013;88(6):884892.

7. Dovey SM, Meyers DS, Phillips RL, Green LA, Fryer GE, Galliher JM, Kappus J, Grob P. A.preliminary taxonomy of medical errors in family practice. BMJ Quality \& Safety. 2002;11(3):233-238.

8. Garosi E, Mazloumi A, Kalantari R, hosseini M. Design and ergonomic evaluation of serum set connector device to the medical solution. JHSW. 2017;7(4):307-318.

9. Hoboubi N, Jahangiri M, Keshavarzi S. Introduction of engineering approach technique in quantitative human error assessment; case study in permit to work system of a petrochemical plant. Iran Occupational Health. 2014;11(5):1-9.

10. Delbari Seyed Ali DSA. Application of Analytical
به صورت كلى در ميان كل وظايف شناسايىشده،

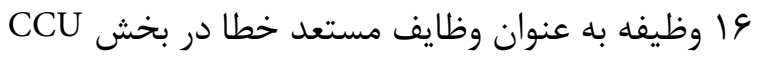
شناسايى شدند، كه وظيفه بيرون كشيدن ترشنات وظحات راههاى هوايى بيشترين ريسك وقوع خطاى انسانى از

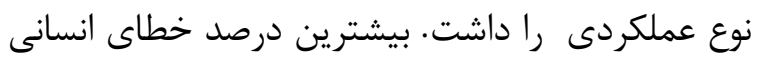

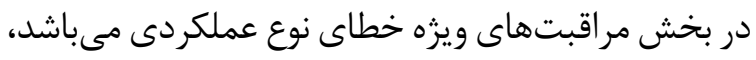

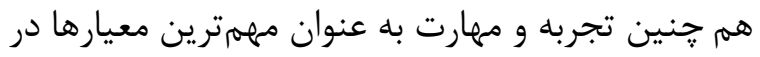

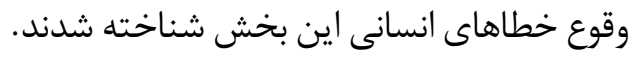

نتيجها نيرى

در بخش مر اقبتهاى ويزه قلب، خطاهاى عملكردى و و احتي

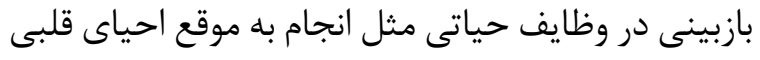

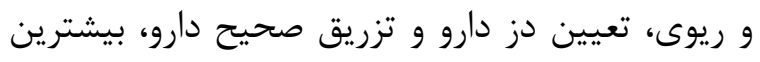

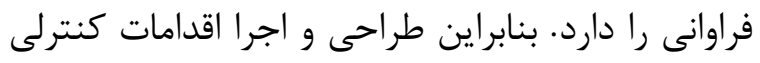

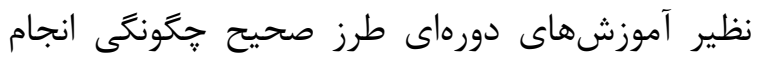

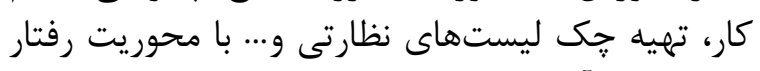

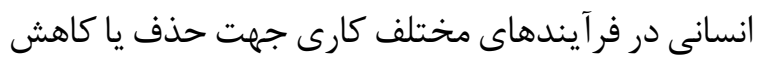

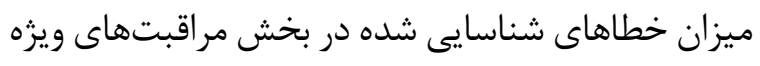

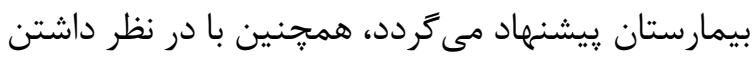

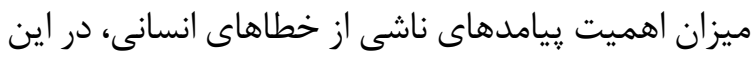

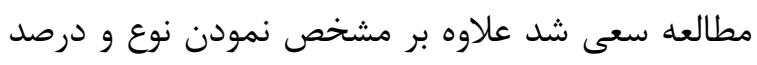

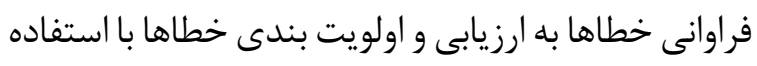
از نظرات خبر گان و فرآيند آيند تحليل سلسله مراتبى (AHP)

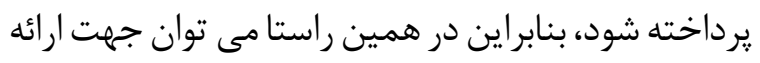

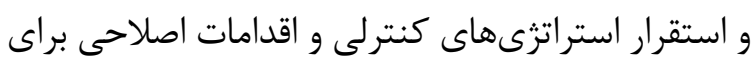
حذف و يا كاهش ريسك ناشى از آنهائر آنها استفاده نمود. محدوديت هاى زيخوهش

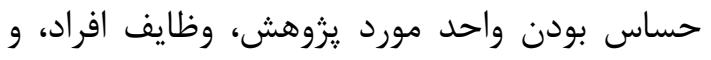

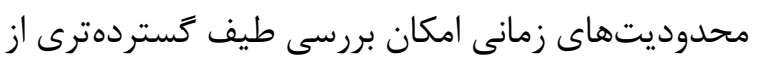

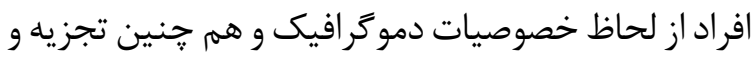

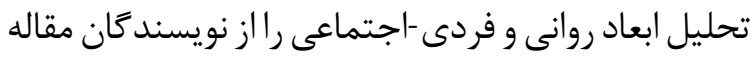

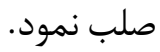

\section{ي بيشنهادات}

ييشنهاد مى گردد كه در مطالعات آتى تمامى ابعاد

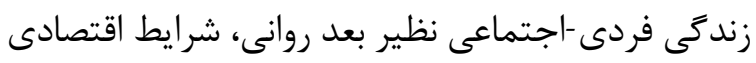

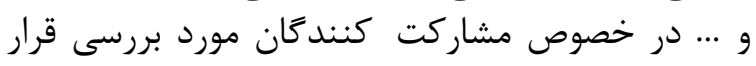

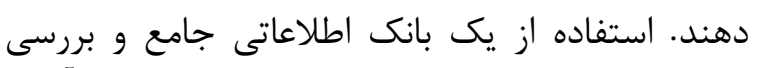

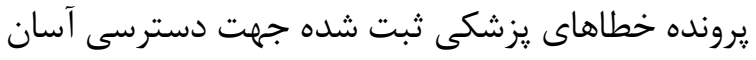

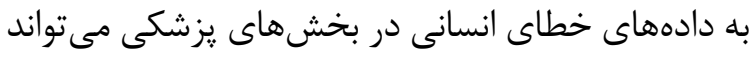

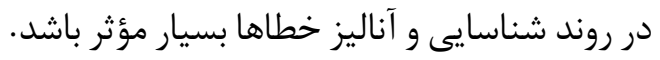


Human Errors in Health Care: Systematic Review. J Saf Promot Inj Prev. 2018; 6(2):87-90. [Persian]

21. Mohammadfam I, Movafagh M, Soltanian A, Salavati M, Bashirian S. Assessment of human errors in the nursing profession of intensive cardiac care unit using SPAR-H method. 2015;7(1):10-22. [Persian]

22. Mohammadfam I, Movafagh M, Bashirian S. Comparison of standardized plant analysis risk human reliability analysis (SPAR-H) and cognitive reliability error analysis meth-ods (CREAM) in quantifying human error in nursing practice. Iranian journal of public health. 2016;45(3):401-402. [Persian]

23. Kazaoka T, Ohtsuka K, Ueno K, Mori M. Why nurses make medication errors: a simulation study. Nurse Education Today. 2007;27(4):312-317.

24. Pourrat X, Antier D, Doucet O, Duchalais A, Lemarié E, Mesny J, Robert M, Meunier P, Rouleau A, Grassin J. Identification and analysis of errors in prescription, preparation and administration of drugs in intensive care, medicine and surgery at the University Hospital Center of Tours. Presse medicale (Paris, France: 1983). 2003 May 1;32(19):876-82.

25. Bagheri-Nesami M, Esmaeili R, Tajari M. Frequency of Non Injectable Medication Administration Errors in Nurses of Cardiac Critical Care Units in Mazandaran Province in 2014. JRUMS. 2016;15(2):151-164. [Persian]

26. Zahmatkeshan N, Bagherzadeh R, Mirzaie K. An observational study to evaluate the medication errors by nursing staff working in Bushehr medical centers during one-year interval. Iran South Med 2010;13(5):201-206. [Persian]

27. Zagheri Tafreshi M, Rassouli M, Zayeri F, Pazookian M. Development of nurses' medication error model: mixed method. Quarterly Journal of Nursing Management. 2014;3(3):35-50. [Persian]

28. Mirzaei M. Khatony A, Safari Faramani R,Sepahvand E. Prevalence and Types of Medicationerrors and Barriers to Reporting Errors by Nursesin an Educational Hospital in Kermanshah. Hayat2013;19(3):28-37. [Persian]
Hierarchy Process Analysis (AHP) technique in the ranking of tourist attractions assessment indicators. 2010;15(2):45-52. [Persian]

11. Babaeipouya A, Mosavianasl Z, Amani S, Moazez Ardebili N. Human error analysis in neonatal intensive care unit by predictive analysis of cognitive errors. J Occup Environ Health. 2017;3(1):38-47. [Persian]

12. Mohsenpour $\mathrm{M}$, Hosseini $\mathrm{M}$, Abbaszadeh $\mathrm{A}$, Shahboulaghi FM, Khankeh H. Nursing error: an integrated review of the literature. Indian journal of medical ethics. 2017;2(2):75-81. [Persian]

13. Ghiyasi S, Heidari M, Hoda A, Azimi L. Human error risk assessment of clinical care in emergency department with SHERPA approach and nurses safety climate analysis. 2018;15(3):129-140. [Persian].

14. Marqui L, Raufaste É, Marin C, Ecoiffier M. Diagnosis Error in Emergency Medicine: Application of the. Le travail humain. 2003;66(4):347-76.

15. Rolston JD, Bernstein M. Errors in neurosurgery. Neurosurgery Clinics. 2015;26(2):149-155.

16. Mohammadfam I, Saeidi C. Evaluating human errors in cataract surgery using the SHERPA technique. J Ergon. 2015; 2(4):41-47. [Persian]

17. Mazlumi A, Kermani A, Nsel seraji J, Ghasemzadeh F. Identification and evaluation of human error using the SHERPA method Emergency practitioners working at Amiralmomenin Hospital in Semnan. Quarterly Journal of Occupational Medicine.2013;5(3):67-78 [Persian]

18. Skinner R, Nelson RR, Chin WW, Land L. The Delphi Method Research Strategy in Studies of Information Systems. CAIS. 2015; 37:2.

19. Asghari M, Nassiri P, Monazzam M R, Golbabaei F, Arabalibeik H, Shamsipour A A et al . Determination and weighting the effective criteria in selecting a heat stress index using the Delphi technique and fuzzy analytic hierarchy process (FAHP). JHSW. 2017;7(1):2332. [Persian]

20. Mohammadfam I, Mohammadi Y, Amiri MR, Karimi S. Identifying and Prioritizing the Factors Affecting on the 